JOURNAL OF INTEGRAL EQUATIONS

AND APPLICATIONS

Volume 6, Number 3, Summer 1994

\title{
PSEUDOSPECTRA AND SINGULAR VALUES OF LARGE CONVOLUTION OPERATORS
}

\author{
ALBRECHT BÖTTCHER
}

\begin{abstract}
This paper is an introduction to $C^{*}$-algebra methods for studying the spectral behavior of large truncated Wiener-Hopf operators. We compute the limit of the norms of the inverses of truncated Wiener-Hopf operators and we show that the pseudospectra (in contrast to the spectra) and the singular values of large truncated Wiener-Hopf operators mimic the pseudospectrum and the singular values of the original operator.
\end{abstract}

1. Introduction. Given a function $k \in L^{1}(\mathbf{R})$ and a finite interval $(\alpha, \beta) \subset \mathbf{R}$, we consider the convolution operator on $L^{2}(\alpha, \beta)$ defined by

$$
\left(W_{\alpha, \beta} \varphi\right)(x)=\int_{\alpha}^{\beta} k(x-t) \varphi(t) d t, \quad \alpha<x<\beta .
$$

What can be said about the spectrum

$$
\Lambda_{0}\left(W_{\alpha, \beta}\right):=\left\{\lambda \in \mathbf{C}: W_{\alpha, \beta}-\lambda I \text { is not invertible }\right\}
$$

if $\tau=\beta-\alpha$ is a large number? In that case one might try one's luck by replacing $W_{\alpha, \beta}$ with the operator $M$ given on $L^{2}(-\infty, \infty)$ by

$$
(M \varphi)(x)=\int_{-\infty}^{\infty} k(x-t) \varphi(t) d t, \quad-\infty<x<\infty,
$$

or since clearly $\Lambda_{0}\left(W_{\alpha, \beta}\right)=\Lambda_{0}\left(W_{0, \tau}\right)$, one could also substitute for $W_{\alpha, \beta}$ the operator $W$ acting on $L^{2}(0, \infty)$ by the rule

$$
(W \varphi)(x)=\int_{0}^{\infty} k(x-t) \varphi(t) d t, \quad 0<x<\infty .
$$

\footnotetext{
Received by the editors on April 14, 1994, and in revised form on June 1, 1994.

Research supported by the Alfried Krupp Förderpreis für junge Hochschullehrer of the Krupp Foundation.

Copyright (C)1994 Rocky Mountain Mathematics Consortium
} 
The spectra of $M$ and $W$ are available: $\Lambda_{0}(M)$ is the closed continuous curve which consists of the origin and the range of the Fourier transform

$$
\hat{k}(\xi)=\int_{-\infty}^{\infty} k(t) e^{i \xi t} d t, \quad-\infty<\xi<\infty
$$

of the kernel $k$, while $\Lambda_{0}(M)$ is the union of the curve $\Lambda_{0}(M)$ and the set of all $\lambda \in \mathbf{C}$ whose winding number (index) with respect to the curve $\Lambda_{0}(M)$ is nonzero. The hopes one has are that the spectrum $\Lambda_{0}\left(W_{\alpha, \beta}\right)$ mimics, in some way, either $\Lambda_{0}(M)$ or $\Lambda_{0}(W)$. To be more precise, given a family $\left\{E_{\tau}\right\}_{\tau>0}$ of sets $E_{\tau} \subset \mathbf{C}$, we denote by $\lim _{\tau \rightarrow \infty} E_{\tau}$ the set of all $\lambda \in \mathbf{C}$ for which there are $\tau_{1}, \tau_{2}, \ldots$ and $\lambda_{1}, \lambda_{2}, \ldots$ such that

$$
0<\tau_{1}<\tau_{2}<\ldots, \quad \tau_{n} \rightarrow \infty, \quad \lambda_{n} \in E_{\tau_{n}}, \quad \lambda_{n} \rightarrow \lambda
$$

and our question then is whether $\lim _{\tau \rightarrow \infty} \Lambda_{0}\left(W_{0, \tau}\right)$ coincides with $\Lambda_{0}(M)$ or $\Lambda_{0}(W)$.

Let us consider two examples. First take $k(t)=e^{-|t|}$. Then $\hat{k}(\xi)=2 /\left(1+\xi^{2}\right)$, hence $\Lambda_{0}(M)=\Lambda_{0}(W)=[0,2]$, and one can indeed show that $\lim _{\tau \rightarrow \infty} \Lambda_{0}\left(W_{0, \tau}\right)=[0,2]$. Secondly, suppose $k(t)=e^{t}$ for $t<0$ and $k(t)=2 e^{-t}$ for $t>0$. Now $\hat{k}(\xi)=(3+i \xi) /\left(1+\xi^{2}\right)$, so $\Lambda_{0}(M)$ is the ellipse

$$
\left\{x+i y \in \mathbf{C}: \frac{(x-3 / 2)^{2}}{(3 / 2)^{2}}+\frac{y^{2}}{(1 / 2)^{2}}=1\right\}
$$

whereas $\Lambda_{0}(W)$ consists of this ellipse and all points in its interior. However, it results that $\lim _{\tau \rightarrow \infty} \Lambda_{0}\left(W_{0, \tau}\right)$ is the union of the circle $\{\lambda \in \mathbf{C}:|\lambda-1 / 2|=1 / 12\}$ and the interval $[3 / 2-\sqrt{2}, 3 / 2+\sqrt{2}]$ (see $[\mathbf{1}]$ and [7]). Thus, while in the first example $\Lambda_{0}\left(W_{\alpha, \beta}\right)=\Lambda_{0}\left(W_{0, \tau}\right)$ mimics both $\Lambda_{0}(M)$ and $\Lambda_{0}(W)$ very well, there is absolutely no relation between $\Lambda_{0}\left(W_{\alpha, \beta}\right)$ and $\Lambda_{0}(M)$ or $\Lambda_{0}(W)$ in the second example.

In the two examples we have examined, the function $\hat{k}(\xi)$ is rational, and one can show that in this case the limit set $\lim _{\tau \rightarrow \infty} \Lambda_{0}\left(W_{0, \tau}\right)$ is always a "thin" set (namely, a finite union of analytic arcs) that is contained in the spectrum $\Lambda_{0}(W)$ of $W$ and that "tries to stay away" from the boundary of $\Lambda_{0}(W)$ (see [19] and [7]). Consequently, if the interior of $\Lambda_{0}(W)$ is not empty, which is the case in the second example, 
then we cannot expect any relation between that limit set and $\Lambda_{0}(W)$ or $\Lambda_{0}(M)$. On the other hand, if, as in the first example, $\Lambda_{0}(W)$ itself is "thin," then there is nothing that might prevent the limit set from being equal to $\Lambda_{0}(W)$.

Things are less clear in case $\hat{k}(\xi)$ is not rational. However, it turns out that whenever we have to deal with self-adjoint operators, there is a close relationship between the limit set of the spectra of the "truncated" operators and the spectrum of the original operator. Here are a few examples that illustrate this observation.

If $k$ is a Hermitian kernel, i.e., if $\overline{k(-t)}=k(t)$, then $\hat{k}$ is real-valued, $W_{0, \tau}$ and $W$ are self-adjoint, and one can show that

$$
\lim _{\tau \rightarrow \infty} \Lambda_{0}\left(W_{0, \tau}\right)=\Lambda_{0}(W)=\left[\inf _{\xi \in \mathbf{R}} \hat{k}(\xi), \sup _{\xi \in \mathbf{R}} \hat{k}(\xi)\right] .
$$

Of course, this equality would also hold with $\Lambda_{0}(M)$ in place of $\Lambda_{0}(W)$. That $W$ is the preferable operator can be motivated as follows. Let us drop for a moment the assumption that $k$ is in $L^{1}(\mathbf{R})$ and consider instead the kernel $k(t)=1 /(\pi i t)$. Then $W_{0, \tau}$ is the Cauchy singular integral operator on $L^{2}(0, \tau)$,

$$
\left(W_{0, \tau} \varphi\right)(x)=\frac{1}{\pi i} \int_{0}^{\tau} \frac{\varphi(t) d t}{t-x}, \quad 0<x<\tau,
$$

and the role of the Fourier transform of $k$ is played by the function $\hat{k}(\xi)=-\operatorname{sign} \xi$. It is well known that in the case at hand $\Lambda_{0}(M)=$ $\{-1,1\}, \Lambda_{0}(W)=[-1,1]$, and $\Lambda_{0}\left(W_{0, \tau}\right)=[-1,1]$ for all $\tau>0$ (see $[\mathbf{1 2}$, pp. $50-51,84-55]$ or $[\mathbf{6}$, p. 416] for the latter equality). Hence, again (1) is valid, but now we cannot replace $\Lambda_{0}(W)$ by $\Lambda_{0}(M)$ in (1). Let finally $k$ be an arbitrary kernel, but consider the singular values of $W_{0, \tau}$ and $W$ instead of their spectra. Recall that the singular values of an operator $T$ are defined as the points in the spectrum of $\left(T^{*} T\right)^{1 / 2}$. The circumstance that $\left(T^{*} T\right)^{1 / 2}$ is self-adjoint nourishes the hope that for large $\tau$ the singular values of $W_{0, \tau}$ mimic the singular values of $W$. This is indeed the case: as will be shown below, we have

$$
\lim _{\tau \rightarrow \infty} \Lambda_{0}\left(\left(W_{0, \tau}^{*} W_{0, \tau}\right)^{1 / 2}\right)=\Lambda_{0}\left(\left(W^{*} W\right)^{1 / 2}\right) \cup \Lambda_{0}\left(\left(W W^{*}\right)^{1 / 2}\right) .
$$

But what about the case where $\hat{k}(\xi)$ is neither rational nor realvalued? In this situation no general results on the limiting behavior 
of $\Lambda_{0}\left(W_{0, \tau}\right)$ are available. However, Reichel and Trefethen [18] have shown us an astonishing way out of the labyrinth. When studying discrete convolution operators, they made a fascinating discovery: the pseudospectra of discrete convolution operators mimic exactly the pseudospectra of an appropriate limiting operator. For $\varepsilon>0$, the $\varepsilon$ pseudospectrum $\Lambda_{\varepsilon}(T)$ of an operator is defined as the set

$$
\Lambda_{\varepsilon}(T)=\left\{\lambda \in \mathbf{C}: \lambda \in \Lambda_{0}(T) \text { or }\left\|(T-\lambda I)^{-1}\right\| \geq 1 / \varepsilon\right\}
$$

We will show that for a large class of kernels $k$

$$
\lim _{\tau \rightarrow \infty} \Lambda_{\varepsilon}\left(W_{0, \tau}\right)=\Lambda_{\varepsilon}(W)
$$

for each $\varepsilon>0$. Thus, passage from spectra to pseudospectra simplifies things drastically: for large $\tau$ the pseudospectrum of $W_{0, \tau}$ mimics the pseudospectrum of $W$, which is in general not true for the "usual" spectrum.

2. Wiener-Hopf operators. The operator $W$ that played a crucial role in the Introduction is a member of the class of Wiener-Hopf operators. The Wiener-Hopf operator $W(a)$ generated by a (complexvalued) function $a \in L^{\infty}(\mathbf{R})$ is the bounded operator on $L^{2}(0, \infty)$ defined by

$$
(W(a) \varphi)(x)=\frac{1}{2 \pi} \int_{-\infty}^{\infty} e^{-i x \xi} a(\xi) \int_{0}^{\infty} e^{i t \xi} \varphi(t) d t d \xi, \quad 0<x<\infty
$$

If $a$ is of the form $a(\xi)=c+\hat{k}(\xi)$ with $c \in \mathbf{C}$ and $k \in L^{1}(\mathbf{R})$, then the action of $W(a)$ may also be given by the formula

$$
(W(a) \varphi)(x)=c \varphi(x)+\int_{0}^{\infty} k(x-t) \varphi(t) d t, \quad 0<x<\infty .
$$

The truncated Wiener-Hopf operator $W_{\tau}(a)$, where $0<\tau<\infty$, is the compression of $W(a)$ to $L^{2}(0, \tau)$, that is, $W_{\tau}(a)=P_{\tau} W(a) \mid L^{2}(0, \tau)$, where $P_{\tau}$ denotes the orthogonal projection of $L^{2}(0, \infty)$ onto $L^{2}(0, \tau)$ :

$$
\left(P_{\tau} \varphi\right)(x)=\varphi(x), \quad 0<x<\tau, \quad\left(P_{\tau} \varphi\right)(x)=0, \quad \tau<x<\infty .
$$


With these notations, the operators $W_{0, \tau}$ and $W$ of the Introduction are just $W_{\tau}(\hat{k})$ and $W(\hat{k})$, respectively. Standard references to the theory of Wiener-Hopf operators are the books $[\mathbf{1 3}, \mathbf{1 1}, \mathbf{1 2}, \mathbf{6}]$.

The function $a$ is usually referred to as the symbol of the operator $W(a)$ and its truncations $W_{\tau}(a)$. It is not the "kernel" $k$ but rather the properties of the symbol $a$ in terms of which one can most easily study the properties of $W(a)$.

Let $C$ denote the collection of all bounded continuous functions $a$ on $\mathbf{R}$ which have finite limits $a(-\infty)$ and $a(+\infty)$ at infinity satisfying $a(-\infty)=a(+\infty)$. The set of all functions $a \in L^{\infty}(\mathbf{R})$ possessing finite one-sided limits $a(\xi-0)$ and $a(\xi+0)$ at each $\xi \in \mathbf{R}$ and having finite limits $a(-\infty)$ and $a(+\infty)$ at infinity is denoted by $P C$. If $a(\xi)=c+\hat{k}(\xi)$ with $c \in \mathbf{C}$ and $k \in L^{1}(\mathbf{R})$, then clearly $a \in C$, but it is well known that not every function in $C$ is of this form. The Cauchy singular integral operator on the half-line is the operator $W(-\operatorname{sign} \xi)$ and is thus the archetypal example of a Wiener-Hopf operator with a piecewise continuous symbol.

Let $a \in P C$. Then $a$ has at most countably many discontinuities (jumps), and we denoted by $a^{\#}$ the closed, continuous, and naturally oriented curve resulting from the essential range of $a$ (= spectrum of $a$ in $L^{\infty}(\mathbf{R})$ ) by filling in line segments between the endpoints of each jump, including the possible jump at infinity. The spectrum $\Lambda_{0}(W(a))$ equals the union of the curve $a^{\#}$ and the set of all points in the plane having nonzero winding number (index) with respect to $a^{\#}$ (see, e.g., [13]). In particular, if even $a \in C$, then $\Lambda_{0}(W(a))$ is the set described in the Introduction.

Now suppose $a \in L^{\infty}(\mathbf{R})$. Then the adjoint operator $W^{*}(a)$ is equal to $W(\bar{a})$ where $\bar{a}(\xi):=\overline{a(\xi)}$. It follows that $W(a)$ is self-adjoint if and only if $a$ is real-valued. One of the oldest results on Wiener-Hopf operators goes back to Brown and Halmos [8]: the operator $W(a)$ is normal, i.e., it commutes with its adjoint, if and only if the essential range $\mathcal{R}(a)$ of $a$ lies on a straight line; in that case the spectrum $\Lambda_{0}(W(a))$ coincides with the line segment $\operatorname{conv} \mathcal{R}(a)$, i.e., with the closed convex hull of $\mathcal{R}(a)$.

We will push the main results to the case of so-called locally normal symbols. The name is motivated by the Brown-Halmos criterion (and was introduced in $[\mathbf{3}]$ ); the consideration of just this class has its reason 
in the fact that it comprises both all piecewise continuous and all realvalued symbols, and the definition of this class is as follows. For an open set $U \subset \mathbf{R}$, denote by $\mathcal{R}_{U}(a)$ the essential values of $a$ on $U$, i.e., the spectrum in $L^{\infty}(U)$ of the restriction of $a$ to $U$. Then for $\xi \in \mathbf{R}$, put

$$
\mathcal{R}_{\xi}(a)=\bigcap_{\varepsilon>0} \mathcal{R}_{(\xi-\varepsilon, \xi+\varepsilon)}(a)
$$

and let

$$
\mathcal{R}_{\infty}(a)=\bigcap_{M>0} \mathcal{R}_{(-\infty,-M) \cup(M, \infty)}(a) .
$$

The function $a$ is said to be locally normal if each of the sets $\mathcal{R}_{\xi}(a)$, $\xi \in \mathbf{R} \cup\{\infty\}$, lies on some straight line (depending on $\xi$ ). In other words, $a$ is locally normal if and only if the convex hull of the "limit values" of $a$ at each point of $\mathbf{R} \cup\{\infty\}$ is some straight line segment. Notice that $\mathcal{R}_{\xi}(a)=\{a(\xi)\}$ if $a \in C$ and $\mathcal{R}_{\xi}(a)=\{a(\xi-0), a(\xi+0)\}$ if $a \in P C$, so that functions in $C$ and $P C$ are automatically locally normal. To have a more complicated example, if $a(\xi)=\sin (1 / \xi)$ in a neighborhood of $0, a(\xi)=i \sin \xi$ in a neighborhood of infinity, and $a$ is continuous on $\mathbf{R} \backslash\{0\}$, then $a$ is locally normal. The set of all locally normal functions will be denoted by $L N$.

3. Finite section method. Given a Hilbert space $H$, we denote by $\mathcal{L}(H)$ the collection of all bounded (linear) operators on $H$. Suppose $A \in \mathcal{L}\left(L^{2}(0, \infty)\right)$ and $\left\{A_{\tau}\right\}_{\tau>0}$ is a family of operators $A_{\tau} \in \mathcal{L}\left(L^{2}(0, \tau)\right)$ such that $A_{\tau} P_{\tau}$ converges strongly (i.e., pointwise) to $A$ as $\tau \rightarrow \infty$. Recall that $P_{\tau}$ is the canonical projection of $L^{2}(0, \infty)$ onto $L^{2}(0, \tau)$. We have this situation if, for example, $A=W(a)$ or $A=W(\bar{a}) W(a)-\lambda I$ and $A_{\tau}=W_{\tau}(a)$ or $A_{\tau}=W_{\tau}(\bar{a}) W_{\tau}(a)-\lambda I$, respectively. Here and in what follows $I$ denotes the identity operator, whatever the space is that it acts upon.

In numerical analysis, we replace the equation $A \varphi=f$ on $L^{2}(0, \infty)$ by the equations $A_{\tau} \varphi_{\tau}=P_{\tau} f$ on $L^{2}(0, \tau)$, assuming that the latter equations are easier to solve than the original equation $A \varphi=f$. We write $A \in \Pi\left\{A_{\tau}\right\}$ if the operators $A_{\tau}$ are invertible on $L^{2}(0, \tau)$ for all sufficiently large $\tau$ and $\varphi_{\tau}=A_{\tau}^{-1} P_{\tau} f$ converges in $L^{2}(0, \infty)$ to a solution of the equation $A \varphi=f$ for every right-hand side $f \in L^{2}(0, \infty)$. If $W(a) \in \Pi\left\{W_{\tau}(a)\right\}$ one says that the finite section method is applicable to $W(a)$. 
Our subject here is not to replace the operator $A$ by the operator $A_{\tau}$, but rather the problem of gaining information about the operators $A_{\tau}$ for large $\tau$ by having recourse to properties of the operator $A$. Hence, let us occupy a more "neutral" position and employ the notion of stable convergence: the family $\left\{A_{\tau}\right\}_{\tau>0}$ is said to be stable if the operators $A_{\tau}$ are invertible for all $\tau$ large enough, for $\tau>\tau_{0}$ say, and if $\sup _{\tau>\tau_{0}}\left\|A_{\tau}^{-1}\right\|<\infty$. On putting $\left\|A_{\tau}^{-1}\right\|=\infty$ in case $A_{\tau}$ is not invertible, we may also define $\left\{A_{\tau}\right\}_{\tau>0}$ to be stable if and only if $\lim \sup _{\tau \rightarrow \infty}\left\|A_{\tau}^{-1}\right\|<\infty$.

Finally, some authors (e.g., [17]) use the concept of the spectrum $P\left\{A_{\tau}\right\}$ of the operator family $\left\{A_{\tau}\right\}_{\tau>0}$. They define $P\left\{A_{\tau}\right\}$ as the set of all $\lambda \in \mathbf{C}$ for which $\lim \sup _{\tau \rightarrow \infty}\left\|\left(A_{\tau}-\lambda I\right)^{-1}\right\|=\infty$.

The next proposition, which is well known (see, e.g., [13] or [6]), shows that all the notions introduced above point to the same thing.

Proposition 3.1. The following are equivalent:

(i) $A \in \Pi\left\{A_{\tau}\right\}$;

(ii) $A$ is invertible and $\left\{A_{\tau}\right\}$ is stable;

(iii) $A$ is invertible and $0 \notin P\left\{A_{\tau}\right\}$.

The finite section method, that is, the problem of deciding whether $W(a) \in \Pi\left\{W_{\tau}(a)\right\}$, has been studied by many people for about 30 years. Since 1987 we know that it is not applicable to every invertible Wiener-Hopf operator:

Theorem $3.2[\mathbf{2 3}]$. There exist $a \in L^{\infty}(\mathbf{R})$ such that $W(a)$ is invertible but $W(a) \notin \Pi\left\{W_{\tau}(a)\right\}$.

On the other hand, the finite section method is applicable to WienerHopf operators with symbols that are not "too bad":

Theorem 3.3. Suppose $a \in L^{\infty}(\mathbf{R})$ is locally normal. Then $W(a) \in \Pi\left\{W_{\tau}(a)\right\}$ if and only if $W(a)$ is invertible. Equivalently,

$$
P\left\{W_{\tau}(a)\right\}=\Lambda_{0}(W(a))
$$


For $a \in P C$ this theorem was already established in the late sixties by Gohberg and Feldman (see [13]). In the form stated here the theorem has never been published, but it is implicit in the book [6, where its discrete version is stated and proved on p. 296 (Theorem 7.32 (iii))].

4. $C^{*}$-algebras: They do a lot of things for us. Theorem 3.3 in conjunction with Proposition 3.1 implies that if $a \in L^{\infty}(\mathbf{R})$ is locally normal and $W(a)$ is invertible, then

$$
\limsup _{\tau \rightarrow \infty}\left\|W_{\tau}^{-1}(a)\right\|<\infty .
$$

However, when studying pseudospectra we need more precise information about the norms $\left\|W_{\tau}^{-1}(a)\right\|$. The best possible thing would be to have the equality

$$
\limsup _{\tau \rightarrow \infty}\left\|W_{\tau}^{-1}(a)\right\|=\left\|W^{-1}(a)\right\|,
$$

and we will really be able to prove this equality. Formulas like (2) cannot be proved by bare hands. The magic wand we will use is $C^{*}$ algebra arguments, and I hope I can convince the reader of the claim made in the heading of the present section. The approach employed in the following goes back to the papers $[\mathbf{2 0}]$ and [4], and it was worked out to a powerful machinery in $[\mathbf{6}]$; our presentation is highly inspired by Silbermann's paper [21].

Let us recall the definition and some relevant properties of $\mathrm{C}^{*}$ algebras (for details and proofs of the facts stated below the reader is referred to [10] and [11]). A map $a \mapsto a^{*}$ of a Banach algebra $A$ into itself is called an involution if

$$
a^{* *}=a, \quad(a+b)^{*}=a^{*}+b^{*}, \quad(a b)^{*}=b^{*} a^{*}, \quad(\lambda a)^{*}=\bar{\lambda} a^{*}
$$

for all $a, b \in A$ and all $\lambda \in$ C. A $C^{*}$-algebra is a Banach algebra $A$ with an involution such that $\|a\|^{2}=\left\|a a^{*}\right\|$ for every $a \in A$. For example, if $X$ is a compact Hausdorff space, then $C(X)$ is a $C^{*}$-algebra with complex conjugation as the involution, or if $H$ is a Hilbert space, then $\mathcal{L}(H)$ is a $C^{*}$-algebra with passage to the adjoint operator as the involution.

A subset of a $C^{*}$-algebra is said to be self-adjoint if it is invariant under the involution. Clearly, every closed and self-adjoint subalgebra 
of a $C^{*}$-algebra is itself a $C^{*}$-algebra. If $A$ is a Banach algebra with unit element and $B$ is a closed subalgebra of $A$ containing the unit element, then for an element $b \in B$ the spectrum in $B$ may be larger than its spectrum in $A$. This does not happen if $A$ is a $C^{*}$-algebra and $B$ is a $C^{*}$-subalgebra of $A$; in that case an element $b \in B$ is invertible in $B$ if (and only if) it is invertible in $A$.

A map $\gamma: A \rightarrow B$ of a $C^{*}$-algebra $A$ to a $C^{*}$-algebra $B$ is referred to as a $C^{*}$-algebra homomorphism if $\gamma$ is a bounded linear operator of $A$ to $B$ that preserves products and involutions. It turns out that the image of a $C^{*}$-algebra homomorphism is always closed (which is not true for an arbitrary Banach algebra homomorphism). A bijective $C^{*}$-algebra homomorphism is called a $C^{*}$-algebra isomorphism. If $A$ is a unital commutative $C^{*}$-algebra with maximal ideal space $X$, then the Gelfand map $\Gamma: A \rightarrow C(X)$ is a $C^{*}$-algebra isomorphism of $A$ onto $C(X)$.

It is well known that the norm of a normal Hilbert space operator coincides with its spectral radius. The same is true in $C^{*}$-algebras with unit element: if $a a^{*}=a^{*} a$, then

$$
\|a\|=r(a):=\max \left\{|\lambda|: \lambda \in \Lambda_{0}(a)\right\} .
$$

The latter equality has a series of remarkable consequences. Here is the one that forms the foundation of our approach to equalities like (2).

Proposition 4.1. If a $C^{*}$-algebra homomorphism preserves spectra, then it also preserves norms. That is, if $\gamma: A \rightarrow B$ is a $C^{*}$ algebra homomorphism of the $C^{*}$-algebra $A$ to the $C^{*}$-algebra $B$ with the property that $\Lambda_{0}(a)=\Lambda_{0}(\gamma(a))$ for every $a \in A$, then $\gamma$ is an isometry, i.e., $\|a\|=\|\gamma(a)\|$ for all $a \in A$.

Proof. Since both $a a^{*}$ and $\gamma(a) \gamma\left(a^{*}\right)$ are normal (even self-adjoint), we have

$$
\begin{aligned}
\|a\|^{2} & =\left\|a a^{*}\right\|=r\left(a a^{*}\right)=r\left(\gamma\left(a a^{*}\right)\right) \\
& =r\left(\gamma(a) \gamma\left(a^{*}\right)\right)=\left\|\gamma(a) \gamma\left(a^{*}\right)\right\|=\|\gamma(a)\|^{2} .
\end{aligned}
$$

Notice also that injective $C^{*}$-algebra homomorphisms automatically preserve spectra and thus norms. Indeed, let $\gamma: A \rightarrow B$ be an injective 
$C^{*}$-algebra homomorphism. Clearly, $\Lambda_{0}(\gamma(a)) \subset \Lambda_{0}(a)$ for every $a \in A$. To get the reverse inclusion, suppose $\gamma(a-\lambda e)$ is invertible in $B$. Since $\gamma(a-\lambda e)$ lies in $\gamma(A)$ and $\gamma(A)$ is a $C^{*}$-subalgebra of $B$, it follows that the inverse of $\gamma(a-\lambda e)$ belongs to $\gamma(A)$ and is therefore of the form $\gamma(c)$ with $c \in A$. The injectivity of $\gamma$ then implies that $c$ is the inverse of $a-\lambda e$.

Finally, given a $C^{*}$-algebra $A$, we may construct new $C^{*}$-algebras as follows. If $J$ is a closed two-sided ideal of $A$, then $J$ is in fact self-adjoint and the quotient algebra $A / J$ is a $C^{*}$-algebra with the involution $(a+J)^{*}:=a^{*}+J$ and the usual quotient norm. Moreover, if $B$ is a $C^{*}$-subalgebra of $A$ and $J$ is a closed two-sided ideal of $A$, then $B+J$ is a $C^{*}$-subalgebra of $A$ and the map

$$
(B+J) / J \rightarrow B /(B \cap J), \quad b+j+J \mapsto b+(B \cap J)
$$

is a well-defined $C^{*}$-algebra isomorphism.

Now let us return to the equality (2). Our first task is to construct a $C^{*}$-algebra whose norm has something to do with the left-hand side of (2). This may be done as follows. Let $\mathfrak{F}$ be the set of all families $\left\{A_{\tau}\right\}_{\tau>0}$ of operators $A_{\tau} \in \mathcal{L}\left(L^{2}(0, \tau)\right)$ such that

$$
\left\|\left\{A_{\tau}\right\}\right\|:=\sup _{\tau>0}\left\|A_{\tau}\right\|<\infty .
$$

With the operations $\left\{A_{\tau}\right\}^{*}=\left\{A_{\tau}^{*}\right\},\left\{A_{\tau}\right\}+\left\{B_{\tau}\right\}=\left\{A_{\tau}+B_{\tau}\right\}$, $\alpha\left\{A_{\tau}\right\}=\left\{\alpha A_{\tau}\right\},\left\{A_{\tau}\right\}\left\{B_{\tau}\right\}=\left\{A_{\tau} B_{\tau}\right\}$, and the norm (3), this set is a $C^{*}$-algebra. To convert the "sup" in (3) into a "limsup," we have recourse to a simple trick: the subset

$$
\mathfrak{N}=\left\{\left\{C_{\tau}\right\} \in \mathfrak{F}:\left\|C_{\tau}\right\| \rightarrow 0 \quad \text { as } \quad \tau \rightarrow \infty\right\}
$$

is a closed two-sided ideal of $\mathfrak{F}$, and if $\left\{A_{\tau}\right\} \in \mathfrak{F}$, then the norm of the coset $\left\{A_{\tau}\right\}+\mathfrak{N}$ in the quotient algebra $\mathfrak{F} / \mathfrak{N}$ is

$$
\left\|\left\{A_{\tau}\right\}+\mathfrak{N}\right\|=\limsup _{\tau \rightarrow \infty}\left\|A_{\tau}\right\| .
$$

Thus, $\mathfrak{F} / \mathfrak{N}$ is the $C^{*}$-algebra we should work in.

The algebra $\mathfrak{F} / \mathfrak{N}$ is too large to be well understood, but it contains nice $C^{*}$-subalgebras. One of them is the smallest $C^{*}$-subalgebra $\mathfrak{A}$ of 
$\mathfrak{F} / \mathfrak{N}$ containing all cosets of the form $\left\{W_{\tau}(a)\right\}+\mathfrak{N}$ with $a \in C$. It is easy to check (see Proposition 5.1 below) that if $\left\{A_{\tau}\right\}+\mathfrak{N} \in \mathfrak{A}$, then the strong limit

$$
S_{1}\left\{A_{\tau}\right\}:=s-\lim _{\tau \rightarrow \infty} A_{\tau}\left(:=s-\lim _{\tau \rightarrow \infty} A_{\tau} P_{\tau}\right)
$$

exists and clearly, $\left\|S_{1}\left\{A_{\tau}\right\}\right\| \leq\left\|\left\{A_{\tau}\right\}+\mathfrak{N}\right\|$. Now denote by $R_{\tau}$ the operator of $L^{2}(0, \infty)$ onto $L^{2}(0, \tau)$ defined by

$$
\left(R_{\tau} \varphi\right)(x)=\overline{\varphi(\tau-x)}, \quad 0<x<\tau, \quad\left(R_{\tau} \varphi\right)(x)=0, \quad x>\tau .
$$

We have $R_{\tau}^{2}=P_{\tau}$ and $R_{\tau} W_{\tau}(a) R_{\tau}=W_{\tau}(\bar{a})$. Using these two equalities one can show (see Proposition 5.1 in the next section) that for every $\left\{A_{\tau}\right\}+\mathfrak{N} \in \mathfrak{A}$ the strong limit

$$
S_{2}\left\{A_{\tau}\right\}:=s-\lim _{\tau \rightarrow \infty} R_{\tau} A_{\tau} R_{\tau}
$$

exists. Again $\left\|S_{2}\left\{A_{\tau}\right\}\right\| \leq\left\|\left\{R_{\tau} A_{\tau} R_{\tau}\right\}+\mathfrak{N}\right\| \leq\left\|\left\{A_{\tau}\right\}+\mathfrak{N}\right\|$. Hence, the mapping

$$
\gamma:\left\{A_{\tau}\right\}+\mathfrak{N} \mapsto\left(S_{1}\left\{A_{\tau}\right\}, S_{2}\left\{A_{\tau}\right\}\right)
$$

is a $C^{*}$-algebra homomorphism of the $C^{*}$-algebra $\mathfrak{A}$ into the $C^{*}$-algebra $\mathcal{L}^{2}:=\mathcal{L}\left(L^{2}(0, \infty)\right) \oplus \mathcal{L}\left(L^{2}(0, \infty)\right)$, the direct sum of two copies of $\mathcal{L}\left(L^{2}(0, \infty)\right)$ with the norm

$$
\|(B, C)\|=\max \{\|B\|,\|C\|\} .
$$

Now suppose we are able to show the following:

$$
\begin{gathered}
\text { for every }\left\{A_{\tau}\right\}+\mathfrak{N} \in \mathfrak{A}, \text { the spectrum of }\left\{A_{\tau}\right\}+\mathfrak{N} \text { in } \mathfrak{A} \\
\text { equals the spectrum of }\left(S_{1}\left(A_{\tau}\right\}, S_{2}\left\{A_{\tau}\right\}\right) \text { in } \mathcal{L}^{2} .
\end{gathered}
$$

Notice that (4) has nothing to do with the exact value of certain norms, it is merely an assertion on stable convergence. Indeed, since $\mathfrak{A}$ is a $C^{*}$-subalgebra of $\mathfrak{F} / \mathfrak{N}$, invertibility in $\mathfrak{A}$ is equivalent to invertibility in $\mathfrak{F} / \mathfrak{N}$, and invertibility in the latter algebra means just stable convergence. Hence, (4) is in fact equivalent to the claim 


$$
\begin{gathered}
\text { if }\left\{A_{\tau}\right\}+\mathfrak{N} \in \mathfrak{A}, \text { then }\left\{A_{\tau}\right\} \text { is stable if and only if } \\
\text { both } S_{1}\left\{A_{\tau}\right\} \text { and } S_{2}\left\{A_{\tau}\right\} \text { are invertible. }
\end{gathered}
$$

Once (5) and thus (4) is shown, we are done; Proposition 4.1 implies that

$$
\left\|\left\{A_{\tau}\right\}+\mathfrak{N}\right\|=\max \left\{\left\|S_{1}\left\{A_{\tau}\right\}\right\|, \| S_{2}\left\{A_{\tau} \|\right\}\right.
$$

for all $\left\{A_{\tau}\right\}+\mathfrak{N} \in \mathfrak{A}$, and in the special case where $A_{\tau}=W_{\tau}^{-1}(a)$ we have in particular

$$
S_{1}\left\{W_{\tau}^{-1}(a)\right\}=W^{-1}(a), \quad S_{2}\left\{W_{\tau}^{-1}(a)\right\}=W^{-1}(\bar{a}),
$$

and since $\left\|W^{-1}(a)\right\|=\left\|W^{-1}(\bar{a})\right\|$, we obtain the equality

$$
\left\|\left\{W_{\tau}^{-1}(a)\right\}+\mathfrak{N}\right\|=\left\|W^{-1}(a)\right\| .
$$

What we are left with is the verification of (5). This requires working with a few more $C^{*}$-algebras.

5. $C^{*}$-algebras in action. Define $\mathfrak{F}, \mathfrak{N}$, and $\mathfrak{A}$ as in the preceding section. In order to study $\mathfrak{A}$, we need to know something about the products $W_{\tau}(b) W_{\tau}(c)$. If $b, c \in C$, then $W_{\tau}(b) W_{\tau}(c)$ is in general not equal to $W_{\tau}(b c)$, but Widom [25] showed that

$$
W_{\tau}(b) W_{\tau}(c)=W_{\tau}(b c)+P_{\tau} K P_{\tau}+R_{\tau} L R_{\tau},
$$

where $K$ and $L$ are compact operators on $L^{2}(0, \infty), P_{\tau}$ is the canonical projection of $L^{2}(0, \infty)$ onto $L^{2}(0, \tau)$, and $R_{\tau}$ is the operator of $L^{2}(0, \infty)$ onto $L^{2}(0, \tau)$ introduced in the previous section. From (6) we infer that $W_{\tau}(b) W_{\tau}(c)$ is equal to $W_{\tau}(c) W_{\tau}(b)$ modulo operators of the form $P_{\tau} K P_{\tau}+R_{\tau} L R_{\tau}$ with $K$ and $L$ compact. This observation motivated Silbermann $[\mathbf{2 0}]$ to check whether the set

$$
\begin{aligned}
& \mathfrak{J}=\left\{\left\{A_{\tau}\right\}+\mathfrak{N} \in \mathfrak{A}:\right. \\
&\left.\qquad A_{\tau}=P_{\tau} K P_{\tau}+R_{\tau} L R_{\tau} \text { with compact } K \text { and } L\right\}
\end{aligned}
$$

is an ideal in $\mathfrak{A}$, and he was in luck: one can indeed show that $\mathfrak{J}$ is a closed two-sided ideal of $\mathfrak{A}$. Consequently, $\mathfrak{A} / \mathfrak{J}$ is a commutative $C^{*}$-algebra! 
But how is invertibility in $\mathfrak{A} / \mathfrak{J}$ related to what we are actually interested in, namely invertibility in $\mathfrak{A}$ ? Here is the answer $[\mathbf{2 0}, \mathbf{6}]$.

Proposition 5.1. Let $\left\{A_{\tau}\right\}+\mathfrak{N} \in \mathfrak{A}$. Then the strong limits $S_{1}\left\{A_{\tau}\right\}$ and $S_{2}\left\{A_{\tau}\right\}$ exist and belong to $\mathcal{L}\left(L^{2}(0, \infty)\right)$. The coset $\left\{A_{\tau}\right\}+\mathfrak{N}$ is invertible in $\mathfrak{A}$ if and only if $S_{1}\left\{A_{\tau}\right\}$ and $S_{2}\left\{A_{\tau}\right\}$ are invertible operators and the coset $\left\{A_{\tau}\right\}+\mathfrak{N}+\mathfrak{J}$ is invertible in $\mathfrak{A} / \mathfrak{J}$.

Proof outline. The collection of all elements of the form

$$
\left\{A_{\tau}\right\}+\mathfrak{N}=\left\{\sum_{j} \prod_{k} W_{\tau}\left(a_{j k}\right)\right\}+\mathfrak{N}, \quad a_{j k} \in C,
$$

the sum and the products finite, is dense in $\mathfrak{A}$. So it suffices to establish the existence of $S_{1}\left\{A_{\tau}\right\}$ and $S_{2}\left\{A_{\tau}\right\}$ for the element of the form (7). But we clearly have

$$
S_{1}\left\{A_{\tau}\right\}=\sum_{j} \prod_{k} W\left(a_{j k}\right),
$$

and since $R_{\tau}^{2}=P_{\tau}$ and $R_{\tau} W_{\tau}(a) R_{\tau}=W_{\tau}(\bar{a})$, it follows that

$$
S_{2}\left\{A_{\tau}\right\}=\sum_{j} \prod_{k} W\left(\bar{a}_{j k}\right) .
$$

Now suppose that $\left\{A_{\tau}\right\}+\mathfrak{N}$ is invertible in $\mathfrak{A}$. Then $\left\{A_{\tau}\right\}+\mathfrak{N}+\mathfrak{J}$ is all the more invertible in $\mathfrak{A} / \mathfrak{J}$. For every $\varphi \in L^{2}(0, \infty)$ and all sufficiently large $\tau$ we have $\|\varphi\| \leq\left\|A_{\tau}^{-1}\right\|\left\|A_{\tau} \varphi\right\|$ and hence $\|\varphi\| \leq$ $M\left\|S_{1}\left\{A_{\tau}\right\} \varphi\right\|$. In the same way one gets $\|\varphi\| \leq M\left\|\left(S_{1}\left(A_{\tau}\right\}\right)^{*} \varphi\right\|$. Consequently, $S_{1}\left\{A_{\tau}\right\}$ is invertible. The invertibility of $S_{2}\left\{A_{\tau}\right\}$ can be shown analogously.

Now assume $S_{1}\left\{A_{\tau}\right\}, S_{2}\left\{A_{\tau}\right\}$, and $\left\{A_{\tau}\right\}+\mathfrak{N}+\mathfrak{J}$ are invertible. Then there is an element $\left\{B_{\tau}\right\}+\mathfrak{N} \in \mathfrak{A}$ such that

$$
A_{\tau} B_{\tau}=I+P_{\tau} K P_{\tau}+R_{\tau} L R_{\tau}+C_{\tau}
$$

with compact operators $K$ and $L$ and $\left\{C_{\tau}\right\} \in \mathfrak{N}$. It follows that

$$
S_{1}\left\{A_{\tau}\right\} S_{1}\left\{B_{\tau}\right\}=I+K, \quad S_{2}\left\{A_{\tau}\right\} S_{2}\left\{B_{\tau}\right\}=I+L,
$$


and hence

$$
M:=\left(S_{1}\left\{A_{\tau}\right\}\right)^{-1}-S_{1}\left\{B_{\tau}\right\}, \quad N:=\left(S_{2}\left\{A_{\tau}\right\}\right)^{-1}-S_{2}\left\{B_{\tau}\right\}
$$

are compact. Put

$$
B_{\tau}^{\prime}=B_{\tau}+P_{\tau} M P_{\tau}+R_{\tau} N R_{\tau}
$$

Then

$$
\begin{aligned}
A_{\tau} B_{\tau}^{\prime}= & I+P_{\tau}\left(K+A_{\tau} P_{\tau} M\right) P_{\tau} \\
& +R_{\tau}\left(L+R_{\tau} A_{\tau} R_{\tau} N\right) R_{\tau}+C_{\tau} \\
= & I+P_{\tau}\left(K+S_{1}\left\{A_{\tau}\right\} M\right) P_{\tau} \\
& +R_{\tau}\left(L+S_{2}\left\{A_{\tau}\right\} N\right) R_{\tau}+C_{\tau}+C_{\tau}^{\prime} \\
= & I+C_{\tau}+C_{\tau}^{\prime}
\end{aligned}
$$

with $\left\{C_{\tau}^{\prime}\right\} \in \mathfrak{N}$, i.e., $\left\{B_{\tau}^{\prime}\right\}+\mathfrak{N}$ is a right inverse of $\left\{A_{\tau}\right\}+\mathfrak{N}$. It can be shown similarly that $\left\{A_{\tau}\right\}+\mathfrak{N}$ is invertible from the left.

The previous proposition reduces the invertibility problem in $\mathfrak{A}$ to studying invertibility in the commutative $C^{*}$-algebra $\mathfrak{A} / \mathfrak{J}$. So our next question reads: what is the maximal ideal space of $\mathfrak{A} / \mathfrak{J}$ ? Denote by $\mathcal{B}$ the smallest $C^{*}$-subalgebra of $\mathcal{L}\left(L^{2}(0, \infty)\right)$ containing all operators $W(a)$ with $a \in C$. One can show (see, e.g., [6, p. 402]) that the set $\mathcal{K}$ of all compact operators on $L^{2}(0, \infty)$ is contained in $\mathcal{B}$ and thus is a closed two-sided ideal of $\mathcal{B}$. So we may consider the quotient algebra $\mathcal{B} / \mathcal{K}$, and this $C^{*}$-algebra is very well understood (see, e.g., [13]), one has

$$
\mathcal{B} / \mathcal{K}=\{W(a)+\mathcal{K}: a \in C\},
$$

$\mathcal{B} / \mathcal{K}$ is commutative, the maximal ideal space may be identified with the one-point compactification $\mathbf{R} \cup\{\infty\}$ of $\mathbf{R}$, and the Gelfand transform $\Gamma$ is given by

$$
\Gamma: \mathcal{B} / \mathcal{K} \rightarrow C(\mathbf{R} \cup\{\infty\}),(\Gamma(W(a)+\mathcal{K}))(\xi)=a(\xi) .
$$

The following result was first established in [4].

Proposition 5.2. For every $\left\{A_{\tau}\right\}+\mathfrak{N} \in \mathfrak{A}$ the strong limit $S_{1}\left\{A_{\tau}\right\}$ belongs to $\mathcal{B}$, and the mapping

$$
\delta: \mathfrak{A} / \mathfrak{J} \rightarrow \mathcal{B} / \mathcal{K}, \quad\left\{A_{\tau}\right\}+\mathfrak{N}+\mathfrak{J} \rightarrow S_{1}\left\{A_{\tau}\right\}+\mathcal{K}
$$


is a well-defined isometric $C^{*}$-algebra isomorphism of $\mathfrak{A} / \mathfrak{J}$ onto $\mathcal{B} / \mathcal{K}$. In particular, $\left\{A_{\tau}\right\}+\mathfrak{N}+\mathfrak{J}$ is invertible in $\mathfrak{A} / \mathfrak{J}$ if and only if $S_{1}\left\{A_{\tau}\right\}$ is a Fredholm operator, i.e., an operator that is invertible modulo compact operators.

Proof outline. Taking into account (6) and the definition of $\mathfrak{J}$ it is readily seen that every element of $\mathfrak{A} / \mathfrak{J}$ is actually of the form $\left\{W_{\tau}(a)\right\}+\mathfrak{N}+\mathfrak{J}$ with $a \in C$. If $K$ and $L$ are compact operators and $\left\{C_{\tau}\right\} \in \mathfrak{N}$, then

$$
W_{\tau}(a)+P_{\tau} K P_{\tau}+R_{\tau} L R_{\tau}+C_{\tau}
$$

converges strongly to $W(a)+K$, hence

$$
\begin{aligned}
\|W(a)+\mathcal{K}\| & \leq\|W(a)+K\| \\
& \leq \liminf _{\tau \rightarrow \infty}\left\|W_{\tau}(a)+P_{\tau} K P_{\tau}+R_{\tau} L R_{\tau}+C_{\tau}\right\|
\end{aligned}
$$

and thus

$$
\|W(a)+\mathcal{K}\| \leq\left\|\left\{W_{\tau}(a)\right\}+\mathfrak{N}+\mathfrak{J}\right\| .
$$

If now $\left\{A_{\tau}\right\}+\mathfrak{N}+\mathfrak{J}=\left\{B_{\tau}\right\}+\mathfrak{N}+\mathfrak{J}$, then there are $a, b \in C$ such that

$$
\left\{A_{\tau}\right\}+\mathfrak{N}+\mathfrak{J}=\left\{W_{\tau}(a)\right\}=\mathfrak{N}+\mathfrak{J},\left\{B_{\tau}\right\}+\mathfrak{N}+\mathfrak{J}=\left\{W_{\tau}(b)\right\}+\mathfrak{N}+\mathfrak{J}
$$

and from (8) we infer that $\|W(a-b)+\mathcal{K}\|=0$. Since there are no nonzero compact Wiener-Hopf operators, it follows that $a=b$, and since

$$
S_{1}\left\{A_{\tau}\right\}=W(a)+K_{1}, \quad S_{1}\left\{B_{\tau}\right\}=W(b)+K_{2}
$$

with certain compact operators $K_{1}$ and $K_{2}$, we obtain that $S_{1}\left\{A_{\tau}\right\}+$ $\mathcal{K}=S_{1}\left\{B_{\tau}\right\}+\mathcal{K}$. This shows that $\delta$ is well-defined. It is immediate from (8) that $\delta$ is continuous. Because $W(a)+\mathcal{K}=S_{1}\left\{W_{\tau}(a)\right\}+\mathcal{K}$, the mapping is surjective, and as $S_{1}\left\{A_{\tau}\right\}+\mathcal{K}$ is always of the form $W(a)+\mathcal{K}$, it follows that $\delta$ is injective. Finally, by virtue of (6), the mapping $\delta$ is a $C^{*}$-algebra homomorphism.

We thus have shown that $\delta$ is a bijective $C^{*}$-algebra homomorphism, and hence it is an isometric $C^{*}$-algebra isomorphism. 
Now we have all that we need to dispose of (5).

Proposition 5.3. Let $\left\{A_{\tau}\right\}+\mathfrak{N} \in \mathfrak{A}$. Then $\left\{A_{\tau}\right\}$ is stable if and only if $S_{1}\left\{A_{\tau}\right\}$ and $S_{2}\left\{A_{\tau}\right\}$ are invertible.

Proof. In view of Proposition 5.1 we are left with showing that $\left\{A_{\tau}\right\}+\mathfrak{N}+\mathfrak{J}$ is automatically invertible in $\mathfrak{A} / \mathfrak{J}$ whenever $S_{1}\left\{A_{\tau}\right\}$ and $S_{2}\left\{A_{\tau}\right\}$ are invertible. In fact, the invertibility of $S_{1}\left\{A_{\tau}\right\}$ is sufficient: if $S_{1}\left\{A_{\tau}\right\}$ is invertible, then so is (all the more) $S_{1}\left\{A_{\tau}\right\}+\mathcal{K}$, and Proposition 5.2 tells us then that $\left\{A_{\tau}\right\}+\mathfrak{N}+\mathfrak{J}$ is also invertible.

Theorem 5.4. Let $\left\{A_{\tau}\right\}+\mathfrak{N} \in \mathfrak{A}$. Then the limit $\lim _{\tau \rightarrow \infty}\left\|A_{\tau}\right\|$ exists and

$$
\lim _{\tau \rightarrow \infty}\left\|A_{\tau}\right\|=\max \left\{\left\|S_{1}\left\{A_{\tau}\right\}\right\|,\left\|S_{2}\left\{A_{\tau}\right\}\right\|\right\}
$$

Proof. Combining Proposition 5.3 with what was said in Section 4, we obtain that

$$
\limsup _{\tau \rightarrow \infty}\left\|A_{\tau}\right\|=\max \left\{\left\|S_{1}\left\{A_{\tau}\right\}\right\|,\left\|S_{2}\left\{A_{\tau}\right\}\right\|\right\} .
$$

Since $A_{\tau}$ converges strongly to $S_{1}\left\{A_{\tau}\right\}$, it follows that

$$
\left\|S_{1}\left\{A_{\tau}\right\}\right\| \leq \liminf _{\tau \rightarrow \infty}\left\|A_{\tau}\right\|,
$$

and because $S_{2}\left\{A_{\tau}\right\}$ is the strong limit of $R_{\tau} A_{\tau} R_{\tau}$, we get

$$
\left\|S_{2}\left\{A_{\tau}\right\}\right\| \leq \liminf _{\tau \rightarrow \infty}\left\|R_{\tau} A_{\tau} R_{\tau}\right\| \leq \liminf _{\tau \rightarrow \infty}\left\|A_{\tau}\right\|
$$

Whew, the last two sections were a hard piece of work. But now we are ready for the harvest...

6. Pseudospectra. In what follows we use the convention that $\left\|T^{-1}\right\|=\infty$ in case $T$ is not invertible. So we may write

$$
\Lambda_{\varepsilon}(T)=\left\{\lambda \in \mathbf{C}:\left\|(T-\lambda I)^{-1}\right\| \geq 1 / \varepsilon\right\} .
$$


Estimating the limit set of the pseudospectra of operator sequences from below leads to the problem whether the resolvent of an operator can have constant norm. The following result shows that this cannot happen. Both this result and its proof are due to Andrzej Daniluk from Cracow (private communication).

Proposition 6.1. Let $H$ be a Hilbert space, and let $T \in \mathcal{L}(H)$. Suppose that $T-\lambda I$ is invertible for all $\lambda$ in some open subset $U$ of $\mathbf{C}$ and $\left\|(T-\lambda I)^{-1}\right\| \leq M$ for all $\lambda \in U$. Then $\left\|(T-\lambda I)^{-1}\right\|<M$ for all $\lambda \in U$.

Proof. A little thought reveals that what we must show is the following: if $U$ is an open subset of $\mathbf{C}$ containing the origin and $\left\|(T-\lambda I)^{-1}\right\| \leq M$ for all $\lambda \in U$, then $\left\|T^{-1}\right\|<M$. To prove this, assume the contrary, i.e., let $\left\|T^{-1}\right\|=M$. We have

$$
(T-\lambda I)^{-1}=\sum_{j=0}^{\infty} \lambda^{j} T^{-j-1}
$$

for all $\lambda$ in some disk $|\lambda| \leq r$. Given any $f \in H$, we therefore get

$$
\left\|(T-\lambda I)^{-1} f\right\|^{2}=\sum_{j, k \geq 0} \lambda^{j} \bar{\lambda}^{k}\left(T^{-j-1} f, T^{-k-1} f\right)
$$

whenever $|\lambda| \leq r$. Integrating the latter equality along the circle $|\lambda|=r$, we obtain

$$
\frac{1}{2 \pi} \int_{0}^{2 \pi}\left\|\left(T-r e^{i \theta} I\right)^{-1} f\right\|^{2} d \theta=\sum_{j=0}^{\infty} r^{2 j}\left\|T^{-j-1} f\right\|^{2},
$$

and since $\left\|\left(T-r e^{i \theta} a I\right)^{-1} f\right\| \leq M\|f\|$, we arrive at the inequality

$$
\left\|T^{-1} f\right\|^{2}+r^{2}\left\|T^{-2} f\right\|^{2} \leq \sum_{j=0}^{\infty} r^{2 j}\left\|T^{-j-1} f\right\|^{2} \leq M^{2}\|f\|^{2} .
$$

Now pick an arbitrary $\varepsilon>0$. Because $\left\|T^{-1}\right\|=M$ by assumption, there is an $f_{\varepsilon} \in H$ such that $\left\|f_{\varepsilon}\right\|=1$ and $\left\|T^{-1} f_{\varepsilon}\right\|^{2}>M^{2}-\varepsilon$. It follows that

$$
M^{2}-\varepsilon+r^{2}\left\|T^{-2} f_{\varepsilon}\right\|^{2}<M^{2},
$$


i.e., $\left\|T^{-2} f_{\varepsilon}\right\|^{2}<\varepsilon r^{-2}$, and consequently,

$$
1=\left\|f_{\varepsilon}\right\|^{2} \leq\left\|T^{2}\right\|^{2}\left\|T^{-2} f_{\varepsilon}\right\|^{2}<\varepsilon r^{-2}\left\|T^{2}\right\|^{2},
$$

which is impossible if $\varepsilon>0$ is sufficiently small. This contradiction shows that $\left\|T^{-1}\right\|<M$.

Now we are in a position to give a lower bound for the limit set of the pseudospectra of the truncations of an arbitrary operator.

Proposition 6.2. Let $A \in \mathcal{L}\left(L^{2}(0, \infty)\right)$ and denote by $A_{\tau}=$ $P_{\tau} A \mid L^{2}(0, \tau)$ the compression of $A$ to $L^{2}(0, \tau)$. Then, for each $\varepsilon>0$,

$$
\Lambda_{\varepsilon}(A) \subset \lim _{\tau \rightarrow \infty} \Lambda_{\varepsilon}\left(A_{\tau}\right) .
$$

Proof. First let $\lambda \in \Lambda_{0}(A)$. We then claim that

$$
\limsup _{\tau \rightarrow \infty}\left\|\left(A_{\tau}-\lambda I\right)^{-1}\right\|=\infty .
$$

Indeed, if $\left\|\left(A_{\tau}-\lambda I\right)^{-1}\right\| \leq M$ for all $\tau>\tau_{0}$, then $\left\|P_{\tau} \varphi\right\| \leq$ $M\left\|\left(A_{\tau}-\lambda I\right) P_{\tau} \varphi\right\|$ for all $\varphi \in L^{2}(0, \infty)$ and passage to the limit $\tau \rightarrow \infty$ gives $\|\varphi\| \leq M\|(A-\lambda I) \varphi\|$ for all $\varphi \in L^{2}(0, \infty)$, implying that the range of $A-\lambda I$ is closed and that $A-\lambda I$ is injective. Considering adjoints we similarly get $\|\varphi\| \leq M\left\|\left(A^{*}-\bar{\lambda} I\right) \varphi\right\|$ for all $\varphi \in L^{2}(0, \infty)$, which shows that the range of $A-\lambda I$ is dense. Hence, if (9) is not valid then $A-\lambda I$ is invertible. Our assumption that $\lambda$ is in $\Lambda_{0}(A)$ therefore implies that (9) holds. From (9) we infer that there is a sequence $\tau_{n} \rightarrow \infty$ such that $\lambda \in \Lambda_{\varepsilon}\left(A_{\tau_{n}}\right)$ and thus $\lambda \in \lim _{\tau \rightarrow \infty} \Lambda_{\varepsilon}\left(A_{\tau}\right)$.

Now suppose that $\lambda \in \Lambda_{\varepsilon}(A) \backslash \Lambda_{0}(A)$. Then $A-\lambda I$ is invertible and $\left\|(A-\lambda I)^{-1}\right\| \geq 1 / \varepsilon$. Let $U$ be any open neighborhood of $\lambda$. From Proposition 6.1 we deduce that there is a $\mu \in U$ such that $\left\|(A-\mu I)^{-1}\right\|>1 / \varepsilon$ and thus $\left\|(A-\mu I)^{-1}\right\| \geq 1 /(\varepsilon-1 / n)$ for all sufficiently large $n$. Consequently, we have $\lambda=\lim _{n \rightarrow \infty} \lambda_{n}$ with $\lambda_{n} \in \Lambda_{\varepsilon-1 / n}(A)$. If $T$ is any invertible Hilbert space operator, then

$$
\left\|T^{-1}\right\|=\sup _{\psi \neq 0} \frac{\left\|T^{-1} \psi\right\|}{\|\psi\|}=\sup _{\varphi \neq 0} \frac{\|\varphi\|}{\|T \varphi\|}=\left(\inf _{\varphi \neq 0} \frac{\|T \varphi\|}{\|\varphi\|}\right)^{-1} .
$$


Hence, for each $n$, there exists a $\varphi$ in $L^{2}(0, \infty)$ such that

$$
\|\varphi\|=1, \quad\left\|\left(A-\lambda_{n} I\right) \varphi\right\|<\varepsilon-1 /(2 n) .
$$

We have

$$
\begin{aligned}
\left\|\left(A_{\tau}-\lambda_{n} I\right) P_{\tau} \varphi\right\|= & \left\|P_{\tau}\left(A-\lambda_{n} I\right) P_{\tau} \varphi\right\| \\
\leq & \left\|\left(A-\lambda_{n} I\right) \varphi\right\| \\
& +\left\|\left(A-\lambda_{n} I\right)\left(I-P_{\tau}\right) \varphi\right\|
\end{aligned}
$$

and since $I-P_{\tau}$ converges strongly to zero, we obtain

$$
\left\|\left(A_{\tau}-\lambda_{n} I\right) P_{\tau} \varphi\right\|<\varepsilon-1 /(3 n)
$$

for all sufficiently large $\tau$. Because $\left\|P_{\tau} \varphi\right\|$ tends to $\|\varphi\|=1$ as $\tau \rightarrow \infty$, we get

$$
\left\|\left(A_{\tau}-\lambda_{n} I\right) P_{\tau} \varphi\right\| /\left\|P_{\tau} \varphi\right\|<\varepsilon-1 /(4 n)<\varepsilon
$$

for all $\tau$ large enough. Now (10) implies that

$$
\left\|\left(A_{\tau}-\lambda_{n} I\right)^{-1}\right\|>1 / \varepsilon
$$

and consequently, $\lambda_{n} \in \Lambda_{\varepsilon}\left(A_{\tau}\right)$ for all sufficiently large $\tau$. This proves that $\lambda=\lim _{n \rightarrow \infty} \lambda_{n}$ belongs to $\lim _{\tau \rightarrow \infty} \Lambda_{\varepsilon}\left(A_{\tau}\right)$.

The previous proof is partially based on arguments of Reddy [17], who stated the inclusion

$$
\Lambda_{\varepsilon}(W(a)) \subset \lim _{\tau \rightarrow \infty} \Lambda_{\varepsilon}\left(W_{\tau}(a)\right)
$$

for $a=c+\hat{k}$ with $c \in \mathbf{C}$ and $k \in L^{1}(\mathbf{R})$.

We now come to upper bounds for the limit set of the pseudospectra of truncated Wiener-Hopf operators.

Theorem 6.3. Let $a \in C$ and suppose that $W(a)$ is invertible. Then $W_{\tau}(a)$ is invertible for all sufficiently large $\tau$, the limit $\lim _{\tau \rightarrow \infty}\left\|W_{\tau}^{-1}(a)\right\|$ exists, and

$$
\lim _{\tau \rightarrow \infty}\left\|W_{\tau}^{-1}(a)\right\|=\left\|W^{-1}(a)\right\|
$$


Proof. Theorem 3.3 implies that $\left\{W_{\tau}(a)\right\}+\mathfrak{N}$ is invertible in $\mathfrak{F} / \mathfrak{N}$ and since $\mathfrak{A}$ is a $C^{*}$-subalgebra of $\mathfrak{F} / \mathfrak{N}$ and $\left\{W_{\tau}(a)\right\}+\mathfrak{N}$ belongs to $\mathfrak{A}$, it follows that $\left\{W_{\tau}^{-1}(a)\right\}+\mathfrak{N}$ also lies in $\mathfrak{A}$. We can thus apply Theorem 5.4 with $A_{\tau}=W_{\tau}^{-1}(a)$. Since

$$
S_{1}\left\{W_{\tau}^{-1}(a)\right\}=W^{-1}(a), \quad S_{2}\left\{W_{\tau}^{-1}(a)\right\}=W^{-1}(\bar{a}),
$$

and $W^{-1}(\bar{a})$ is nothing but the adjoint of $W^{-1}(a)$, we arrive at (11). 口

Theorem 6.4. If $a \in C$ and $\varepsilon>0$, then

$$
\lim _{\tau \rightarrow \infty} \Lambda_{\varepsilon}\left(W_{\tau}(a)\right)=\Lambda_{\varepsilon}(W(a))
$$

Proof. By virtue of Proposition 6.2, we are left with showing that the left-hand side of (12) is a subset of the right-hand side. So let $\lambda \notin \Lambda_{\varepsilon}(W(a))$. Then $W(a)-\lambda I=W(a-\lambda)$ is invertible and

$$
\left\|W^{-1}(a-\lambda)\right\|=1 / \varepsilon-2 \delta<1 / \varepsilon
$$

Theorem 6.3 implies that there is a $\tau_{0}>0$ such that $W_{\tau}(a-\lambda)$ is invertible and

$$
\left\|W_{\tau}^{-1}(a-\lambda)\right\|<1 / \varepsilon-\delta
$$

for all $\tau>\tau_{0}$. If $\tau>\tau_{0}$ and $|\mu-\lambda|<\varepsilon \delta(1 / \varepsilon-\delta)^{-1}$, then

$$
\begin{aligned}
\left\|W_{\tau}^{-1}(a-\mu)\right\| & \leq \frac{\left\|W_{\tau}^{-1}(a-\lambda)\right\|}{1-|\mu-\lambda|\left\|W_{\tau}^{-1}(a-\lambda)\right\|} \\
& <\frac{1 / \varepsilon-\delta}{1-\varepsilon \delta(1 / \varepsilon-\delta)^{-1}(1 / \varepsilon-\delta)} \\
& =1 / \varepsilon
\end{aligned}
$$

and hence $\mu \notin \Lambda_{\varepsilon}\left(W_{\tau}(a)\right)$. $\quad \square$

For "triangular" Wiener-Hopf operators, that is, for symbols of the form $a_{+}=c+\hat{k}_{+}$or $a_{-}=c+\hat{k}_{-}$with $c \in \mathbf{C}, k_{+} \in L^{1}(0, \infty)$, 
$k_{-} \in L^{1}(-\infty, 0)$, Theorem 6.4 was already proved in [17]. In this case Theorem 6.3 is trivial, because then

$$
\begin{aligned}
\left\|W_{\tau}^{-1}\left(a_{ \pm}\right)\right\| & =\left\|W_{\tau}\left(a_{ \pm}^{-1}\right)\right\| \rightarrow\left\|W\left(a_{ \pm}^{-1}\right)\right\| \\
& =\left\|W^{-1}\left(a_{ \pm}\right)\right\| \quad \text { as } \tau \rightarrow \infty ;
\end{aligned}
$$

moreover, in this case we have

$$
\left\|W^{-1}\left(a_{ \pm}-\lambda\right)\right\|=\left\|\left(a_{ \pm}-\lambda\right)^{-1}\right\|_{L^{\infty}(\mathbf{R})}=1 / \operatorname{dist}\left(\lambda, a_{ \pm}(\mathbf{R} \cup\{\infty\})\right)
$$

for every $\lambda \in \mathbf{C} \backslash \Lambda_{0}\left(W\left(a_{ \pm}\right)\right)$, which implies that

$$
\Lambda_{\varepsilon}^{0}\left(W\left(a_{ \pm}\right)\right)=\Lambda_{\varepsilon}\left(W\left(a_{ \pm}\right)\right)=\left\{\lambda \in \mathbf{C}: \operatorname{dist}\left(\lambda, \Lambda_{0}\left(W\left(a_{ \pm}\right)\right)\right) \leq \varepsilon\right\} .
$$

7. Singular values. Recall that $\mathfrak{A}$ stands for the smallest $C^{*}$ subalgebra of $\mathfrak{F} / \mathfrak{N}$ containing all cosets of the form $\left\{W_{\tau}(a)\right\}+\mathfrak{N}$ with $a \in C$. For $\left\{A_{\tau}\right\}+\mathfrak{N} \in \mathfrak{A}$, we put, as above,

$$
S_{1}\left\{A_{\tau}\right\}=s-\lim _{\tau \rightarrow \infty} A_{\tau}, \quad S_{2}\left\{A_{\tau}\right\}=s-\lim _{\tau \rightarrow \infty} R_{\tau} A_{\tau} R_{\tau} .
$$

Theorem 7.1. Let $\left\{A_{\tau}\right\}+\mathfrak{N} \in \mathfrak{A}$. Then $\left\{A_{\tau}\right\}$ is stable if and only if $S_{1}\left\{A_{\tau}\right\}$ and $S_{2}\left\{A_{\tau}\right\}$ are invertible. In that case the limit $\lim _{\tau \rightarrow \infty}\left\|A_{\tau}^{-1}\right\|$ exists and

$$
\lim _{\tau \rightarrow \infty}\left\|A_{\tau}^{-1}\right\|=\max \left\{\left\|\left(S_{1}\left\{A_{\tau}\right\}\right)^{-1}\right\|,\left\|\left(S_{2}\left\{A_{\tau}\right\}\right)^{-1}\right\|\right\} .
$$

Proof. The first assertion is Proposition 5.3. If $\left\{A_{\tau}\right\}$ is stable, then $\left\{A_{\tau}^{-1}\right\}+\mathfrak{N} \in \mathfrak{A}$ and Theorem 5.4 implies that $\lim _{\tau \rightarrow \infty}\left\|A_{\tau}^{-1}\right\|$ equals the maximum of

$$
\left\|S_{1}\left\{A_{\tau}^{-1}\right\}\right\|=\left\|\left(S_{1}\left\{A_{\tau}\right\}\right)^{-1}\right\| \quad \text { and } \quad\left\|S_{2}\left\{A_{\tau}^{-1}\right\}\right\|=\left\|\left(S_{2}\left\{A_{\tau}\right\}\right)^{-1}\right\| .
$$

\section{Example 7.2. Let}

$$
A_{\tau}=\sum_{j} \prod_{k} W_{\tau}\left(a_{j k}\right), \quad a_{j k} \in C
$$


where the sum and the products are finite. We have

$$
S_{1}\left\{A_{\tau}\right\}=\sum_{j} \prod_{k} W\left(a_{j k}\right), \quad S_{2}\left\{A_{\tau}\right\}=\sum_{j} \prod_{k} W\left(\overline{a_{j k}}\right) .
$$

Theorem 7.1 implies that if both $S_{1}\left\{A_{\tau}\right\}$ and $S_{2}\left\{A_{\tau}\right\}$ are invertible, then so is $A_{\tau}$ for all sufficiently large $\tau$ and

$$
\begin{aligned}
\lim _{\tau \rightarrow \infty} \| & \left(\sum_{j} \prod_{k} W_{\tau}\left(a_{j k}\right)\right)^{-1} \| \\
& =\max \left\{\left\|\left(\sum_{j} \prod_{k} W\left(a_{j k}\right)\right)^{-1}\right\|,\left\|\left(\sum_{j} \prod_{k} W\left(\overline{a_{j k}}\right)\right)^{-1}\right\|\right\} .
\end{aligned}
$$

Theorem 7.3. Let $\left\{A_{\tau}\right\}+\mathfrak{N} \in \mathfrak{A}$ and suppose $A_{\tau}$ is self-adjoint for every $\tau>0$. Then

$$
\lim _{\tau \rightarrow \infty} \Lambda_{0}\left(A_{\tau}\right)=\Lambda_{0}\left(S_{1}\left\{A_{\tau}\right\}\right) \cup \Lambda_{0}\left(S_{2}\left\{A_{\tau}\right\}\right) .
$$

Proof. The operators $A_{\tau}, S_{1}\left\{A_{\tau}\right\}, S_{2}\left\{A_{\tau}\right\}$ are all self-adjoint and hence all spectra in (13) are real. So let $\lambda \in \mathbf{R}$.

If $\lambda \notin \lim _{\tau \rightarrow \infty} \Lambda_{0}\left(A_{\tau}\right)$, then there are a $\delta>0$ and a $\tau_{0}>0$ such that $|\lambda-\mu|>\delta$ for all $\mu \in \cup\left\{\Lambda_{0}\left(A_{\tau}\right): \tau>\tau_{0}\right\}$. It follows that $A_{\tau}-\lambda I$ is invertible for all $\tau>\tau_{0}$ and that

$$
\sup _{\tau>\tau_{0}}\left\|\left(A_{\tau}-\lambda I\right)^{-1}\right\|=\sup _{\tau>\tau_{0}} \max _{\mu \in \Lambda_{0}\left(A_{\tau}\right)} \frac{1}{|\lambda-\mu|} \leq \frac{1}{\delta}
$$

(note that $\left(A_{\tau}-\lambda I\right)^{-1}$ is self-adjoint). On the other hand, if (14) holds then $|\lambda-\mu| \geq \delta$ for all $\mu \in \cup\left\{\Lambda_{0}\left(A_{\tau}\right): \tau>\tau_{0}\right\}$, which implies that $\lambda \notin \lim _{\tau \rightarrow \infty} \Lambda_{0}\left(A_{\tau}\right)$. Consequently, we have

$$
\lambda \notin \lim _{\tau \rightarrow \infty} \Lambda_{0}\left(A_{\tau}\right) \Longleftrightarrow \limsup _{\tau \rightarrow \infty}\left\|\left(A_{\tau}-\lambda I\right)^{-1}\right\|<\infty .
$$

Now Theorem 7.1 can be used to conclude that $\lambda \notin \lim _{\tau \rightarrow \infty} \Lambda_{0}\left(A_{\tau}\right)$ if and only if $S_{1}\left\{A_{\tau}\right\}-\lambda I$ and $S_{2}\left\{A_{\tau}\right\}-\lambda I$ are invertible, which gives the assertion. 
Example 7.4. If $a \in C$ is real-valued, then $W_{\tau}(a)$ as well as $S_{1}\left\{W_{\tau}(a)\right\}=W(a)$ and $S_{2}\left\{W_{\tau}(a)\right\}=W(\bar{a})=W(a)$ are self-adjoint. Theorem 7.3 with $A_{\tau}=W_{\tau}(a)$ gives

$$
\lim _{\tau \rightarrow \infty} \Lambda_{0}\left(W_{\tau}(a)\right)=\Lambda_{0}(W(a)) .
$$

Note that in fact the latter equality can easily be shown to hold for every real-valued $a \in L^{\infty}$ (see, e.g., [7]).

Example 7.5. Let $A_{\tau}$ be as in Example 7.2, but now suppose that all the functions $a_{j k}$ are real-valued. Theorem 7.3 implies that

$$
\lim _{\tau \rightarrow \infty} \Lambda_{0}\left(\sum_{j} \prod_{k} W_{\tau}\left(a_{j k}\right)\right)=\Lambda_{0}\left(\sum_{j} \prod W\left(a_{j k}\right)\right) .
$$

Example 7.6. Let $a \in C$ be any (not necessarily real-valued) function and apply Theorem 7.3 with $A_{\tau}=W_{\tau}(\bar{a}) W_{\tau}(a)$. It results that

$$
\lim _{\tau \rightarrow \infty} \Lambda_{0}\left(W_{\tau}(\bar{a}) W_{\tau}(a)\right)=\Lambda_{0}(W(\bar{a}) W(a)) \cup \Lambda_{0}(W(a) W(\bar{a}))
$$

and hence, by the spectral mapping theorem,

$$
\lim _{\tau \rightarrow \infty} \Lambda_{0}\left(f\left(W_{\tau}(\bar{a}) W_{\tau}(a)\right)\right)=\Lambda_{0}(f(W(\bar{a}) W(a))) \cup \Lambda_{0}(f(W(a) W(\bar{a})))
$$

for every continuous function $f$ on $\mathbf{R}$. In the case where $f(\lambda)=|\lambda|^{1 / 2}$ we obtain a result on the limit set of the singular values of $W_{\tau}(a)$ :

$$
\begin{aligned}
\lim _{\tau \rightarrow \infty} \Lambda_{0}\left(\left(W_{\tau}(\bar{a}) W_{\tau}(a)\right)^{1 / 2}\right) & \\
= & \Lambda_{0}\left((W(\bar{a}) W(a))^{1 / 2}\right) \cup \Lambda_{0}\left((W(a) W(\bar{a}))^{1 / 2}\right)
\end{aligned}
$$

8. Discontinuous symbols. Convolutions with $L^{1}$ kernels lead to Wiener-Hopf operators with continuous symbols. Wiener-Hopf operators with discontinuous symbols arise when studying convolutions 
with less harmless kernels. If, for example, $a(\xi)=-\operatorname{sign}(\xi-\alpha)$, then $W(a)$ may be given on $L^{2}(0, \infty)$ by

$$
(W(a) \varphi)(x)=\frac{1}{\pi i} \int_{0}^{\infty} \frac{e^{i \alpha(t-x)}}{t-x} \varphi(t) d t, \quad x>0,
$$

the integral understood in the sense of the Cauchy principal value. Hence, piecewise constant symbols produce convolutions with kernels of the form $k(t)=p(t) / t$, where $p(t)$ is an almost periodic polynomial. If the kernel is $k(t)=p(t) / t+l(t)$ with an almost periodic polynomial $p(t)$ and an $L^{1}$ function $l(t)$, then the symbol is no longer piecewise constant but still piecewise continuous. Finally, we remark that the class of Wiener-Hopf operators with discontinuous symbols also comprises the compressions of difference operators to the half-line. For instance, if $a(\xi)=\cos \xi$, then $W(a)$ acts on $L^{2}(0, \infty)$ by the rule

$$
(W(a) \varphi)(x)= \begin{cases}(\varphi(x-1)+\varphi(x+1)) / 2 & \text { for } x>1, \\ (\varphi(x+1)) / 2 & \text { for } 0<x<1,\end{cases}
$$

that is, $W(a)$ is convolution by $(\delta(t+1)+\delta(t-1)) / 2$ compressed to the half-line.

It turns out that many of the results established in the preceding sections remain literally valid for operators with piecewise continuous or even locally normal symbols (for the definition of local normality recall Section 2).

In Section 4 we introduced the $C^{*}$-algebra $\mathfrak{A}$ as the smallest $C^{*}$ subalgebra of $\mathfrak{F} / \mathfrak{N}$ containing all cosets $\left\{W_{\tau}(a)\right\}+\mathfrak{N}$ with $a \in C$. Now let $\mathfrak{C}$ denote the smallest closed $C^{*}$-subalgebra of $\mathfrak{F} / \mathfrak{N}$ containing all cosets $\left\{W_{\tau}(a)\right\}+\mathfrak{N}$ with $a \in P C$, and given a locally normal function $a \in L N$, denote by $\mathfrak{D}_{a}$ the smallest closed $C^{*}$-subalgebra of $\mathfrak{F} / \mathfrak{N}$ containing the coset $\left\{W_{\tau}(a)\right\}+\mathfrak{N}$ and all cosets $\left\{W_{\tau}(c)\right\}+\mathfrak{N}$ with $c \in C$.

Theorem 8.1. Theorems 6.3 and 6.4 as well as all assertions made in Examples 7.2, 7.5 and 7.6 are true with $C$ replaced by PC. Theorems 7.1 and 7.3 remain valid with $\mathfrak{A}$ replaced by $\mathfrak{C}$.

Theorem 8.2. Theorems 6.3 and 6.4 and the limit formula established in Example 7.6 are valid with $C$ replaced by $L N$. If $a \in L N$, 
then Theorems 7.1 and 7.3 as well as the conclusions of Examples 7.2 and 7.5 hold with $\mathfrak{D}_{a}$ in place of $\mathfrak{A}$.

The proofs of the last two theorems are based on a combination of the $C^{*}$-machinery developed in Sections 4 and 5 with the usage of so-called local principles. I will not give these proofs in detail here, but I will try to single out the main steps and ideas.

Let us first consider the $P C$ case. All that was said in Section 4 remains valid with $\mathfrak{A}$ replaced by $\mathfrak{C}$, and so we can enter Section 5 . The first problem comes about with Widom's formula (6): if $b, c \in P C$ then this formula holds, but the operators $K$ and $L$ are in general no longer compact. Nevertheless, it turns out that the set

$$
\begin{aligned}
\mathfrak{J}=\left\{\left\{A_{\tau}\right\}+\mathfrak{N} \in \mathfrak{C}:\right. & A_{\tau} \\
& \left.=P_{\tau} K P_{\tau}+R_{\tau} L R_{\tau} \text { with compact } K \text { and } L\right\}
\end{aligned}
$$

is a closed two-sided ideal of $\mathfrak{C}$ and that, moreover, $\mathfrak{C} / \mathfrak{J}$ is a commutative $C^{*}$-algebra.

I want at least to indicate why $\mathfrak{C} / \mathfrak{J}$ is commutative. To simplify notation, we write $A_{\tau} \equiv B_{\tau}$ if $\left\{A_{\tau}\right\}+\mathfrak{N}$ and $\left\{B_{\tau}\right\}+\mathfrak{N}$ are in $\mathfrak{C} / \mathfrak{N}$ and $\left\{A_{\tau}\right\}+\mathfrak{N}+\mathfrak{J}=\left\{B_{\tau}\right\}+\mathfrak{N}+\mathfrak{J}$. We must show that $W_{\tau}(b) W_{\tau}(c) \equiv W_{\tau}(c) W_{\tau}(b)$ whenever $b, c \in P C$. A little thought reveals that we may confine ourselves to the case where $b$ and $c$ have only one jump, at $\beta$ and $\gamma$, say. If $b$ and $c$ have the jump at distinct points, i.e., if $\beta \neq \gamma$, then the operators $K$ and $L$ in (6) are compact and hence

$$
W_{\tau}(b) W_{\tau}(c) \equiv W_{\tau}(b c)=W_{\tau}(c b) \equiv W_{\tau}(c) W_{\tau}(b)
$$

in this case. So assume $\beta=\gamma$. Then there is a constant $\lambda \in \mathbf{C}$ and a function $f \in C$ such that $b=\lambda c+f$. It follows that

$$
\begin{aligned}
W_{\tau}(b) & W_{\tau}(c)-W_{\tau}(c) W_{\tau}(b) \\
& =W_{\tau}(\lambda c+f) W_{\tau}(c)-W_{\tau}(c) W_{\tau}(\lambda c+f) \\
& =W_{\tau}(f) W_{\tau}(C)-W_{\tau}(c) W_{\tau}(f)
\end{aligned}
$$

and since now $f$ and $c$ have no common discontinuity, we obtain

$$
W_{\tau}(f) W_{\tau}(c)-W_{\tau}(c) W_{\tau}(f) \equiv 0 .
$$


This proves that $\mathfrak{C} / \mathfrak{J}$ is commutative.

Let us proceed further. Proposition 5.1 holds with $\mathfrak{A}$ replaced by $\mathfrak{C}$, and so we are left with finding the maximal ideal space of $\mathfrak{C} / \mathfrak{J}$. Denote by $\mathcal{C}$ the smallest $C^{*}$-subalgebra of $\mathcal{L}\left(L^{2}(0, \infty)\right)$ containing all operators $W(a)$ with $a \in P C$. The structure of the quotient algebra $\mathcal{C} / \mathcal{K}$ was uncovered by Gohberg and Krupnik [14]. They showed that the $C^{*}$-algebra $\mathcal{C} / \mathcal{K}$ is commutative, that its maximal ideal space may be identified with the cylinder

$$
M=(\mathbf{R} \cup\{\infty\}) \times[0,1]
$$

(with an exotic topology), and that the Gelfand transform of $W(a)+\mathcal{K}$ is for $a \in P C$ given by

$$
(\Gamma(W(a)+\mathcal{K}))(\xi, \mu)=a(\xi-0)(1-\mu)+a(\xi+0) \mu .
$$

Suppose now that we are able to show the following analogue of Proposition 5.2: the mapping

$$
\delta: \mathfrak{C} / \mathfrak{J} \rightarrow \mathcal{C} / \mathcal{K}, \quad\left\{A_{\tau}\right\}+\mathfrak{N}+\mathfrak{J} \mapsto S_{1}\left\{A_{\tau}\right\}+\mathcal{K}
$$

is a well-defined isometric $C^{*}$-algebra isomorphism between $\mathfrak{C} / \mathfrak{J}$ and $\mathcal{C} / \mathcal{K}$. Then we would get Proposition 5.3 and Theorem 5.4 with $\mathfrak{A}$ replaced by $\mathfrak{C}$, and after that all assertions of Theorem 8.1 could be proved exactly as in Sections 6 and 7.

That $\mathfrak{C} / \mathfrak{J}$ and $\mathcal{C} / \mathcal{K}$ are really isometrically isomorphic was first shown in [4]. Here is a sketch of a proof (different from the original one in [4]). It is relatively easy to see that $\delta: \mathfrak{C} / \mathfrak{J} \rightarrow \mathcal{C} / \mathcal{K}$ is a well-defined $C^{*}$-homomorphism of $\mathfrak{C} / \mathfrak{J}$ onto $\mathcal{C} / \mathcal{K}$. To show that $\delta$ preserves spectra and is thus isometric, we proceed as in Section 4: we prove that

$$
\begin{aligned}
& \text { if }\left\{A_{\tau}\right\}+\mathfrak{N}+\mathfrak{J} \in \mathfrak{C} / \mathfrak{J} \text {, then }\left\{A_{\tau}\right\}+\mathfrak{N}+\mathfrak{N} \text { is invertible } \\
& \text { in } \mathfrak{C} / \mathfrak{J} \text { if and only if } S_{1}\left\{A_{\tau}\right\}+\mathcal{K} \text { is invertible in } \mathcal{C} / \mathcal{K} \text {. }
\end{aligned}
$$

To show (15) we apply the local principle of Allan and Douglas (see, e.g., $[\mathbf{1 1}]$ or $[\mathbf{6}])$. This local principle says the following. Let $R$ be a $C^{*}$-algebra, and let $Z$ be a $C^{*}$-subalgebra of the center of $R$. Denote 
the maximal ideal space of $Z$ by $N$, and for a maximal ideal $m \in N$, let $Y_{m}$ denote the smallest closed two-sided ideal of $R$ containing $m$. Then an element $a \in R$ is invertible in $R$ if and only if the cosets $a+Y_{m}$ are invertible in $R / Y_{m}$ for all $m \in N$.

Put $R=\mathfrak{C} / \mathfrak{J}$. Taking into account that (6) holds with compact $K$ and $L$ if at least one of the functions $b$ and $c$ is continuous and defining $\mathfrak{A}$ as in Section 4 , we see that $Z=(\mathfrak{A}+\mathfrak{J}) / \mathfrak{J}$ is a $C^{*}$-subalgebra of the center of $R$. Proposition 5.1 tells us that the maximal ideal space of $(\mathfrak{A}+\mathfrak{J}) / \mathfrak{J} \cong \mathfrak{A} /(\mathfrak{A} \cap \mathfrak{J})$ is $\mathbf{R} \cup\{\infty\}$ (notice that the $\mathfrak{J}$ in Proposition 5.1 is not the $\mathfrak{J}$ employed here but is equal to $\mathfrak{A} \cap \mathfrak{J}$ ). Now fix $m \in \mathbf{R} \cup\{\infty\}$, and for the sake of simplicity, assume $m \in \mathbf{R}$. The ideal $\mathfrak{Y}_{m}$ is the smallest closed two-sided ideal of $\mathfrak{C} / \mathfrak{J}$ containing all cosets $\left\{W_{\tau}(\varphi)\right\}+\mathfrak{N}+\mathfrak{J}$ such that $\varphi \in P C$ is continuous at $m$ and vanishes there. For $a \in P C$, define $a_{m} \in P C$ by

$$
a_{m}(\xi)=a(m-0)\left(1-\chi_{m}^{+}(\xi)\right)+a(m+0) \chi_{m}^{+}(\xi),
$$

where $\chi_{m}^{+}$is the characteristic function of the interval $(m, \infty)$. Then $a-a_{m}$ is continuous at $m$ and $\left(a-a_{m}\right)(m)=0$. Hence,

$$
\left\{W_{\tau}(a)\right\}+\mathfrak{N}+\mathfrak{J}+\mathfrak{Y}_{m}=\left\{W_{\tau}\left(a_{m}\right)\right\}+\mathfrak{N}+\mathfrak{J}+\mathfrak{Y}_{m}
$$

and we have replaced $\left\{W_{\tau}(a)\right\}$ with general $a \in P C$ by $\left\{W_{\tau}\left(a_{m}\right)\right\}$, where $a_{m}$ is a linear combination of 1 (the function identically 1 ) and $\chi_{m}^{+}$. Since $R=\mathfrak{C} / \mathfrak{J}$ is generated by $\left\{W_{\tau}(a)\right\}+\mathfrak{N}+\mathfrak{J}$ with $a \in P C$, it follows that $R / \mathfrak{Y}_{m}=(\mathfrak{C} / \mathfrak{J}) / \mathfrak{Y}_{m}$ is generated by the identity and by

$$
\left\{W_{\tau}\left(\chi^{+}\right)\right\}+\mathfrak{N}+\mathfrak{J}+\mathfrak{Y}_{m} .
$$

The spectrum of the element (16) can be shown to be the interval $[0,1]$, and therefore $R / \mathfrak{Y}_{m}$ is isometrically isomorphic to $C[0,1]$. For any $\left\{A_{\tau}\right\}+\mathfrak{N}+\mathfrak{J} \in \mathfrak{C} / \mathfrak{J}$, the element

$$
\left\{A_{\tau}\right\}+\mathfrak{N}+\mathfrak{J}+\mathfrak{Y}_{m} \in(\mathfrak{C} / \mathfrak{J}) / \mathfrak{Y}_{m}
$$

may therefore be represented by a well-defined function (the "local representative") in $C[0,1]$.

Now repeat the construction of the preceding paragraph, but this time with $R=\mathcal{C} / \mathcal{K}$ and $Z=\mathcal{B} / \mathcal{K}$, where $\mathcal{B}$ is as in Section 5 . What results is that

$$
S_{1}\left\{A_{\tau}\right\}+\mathcal{K}+\mathcal{Y}_{m} \in(\mathcal{B} / \mathcal{K}) / \mathcal{Y}_{m}
$$


is represented by the same function in $C[0,1]$ as (17). Thus, the spectra of the elements (17) and (18) coincide. The local principle says that $\left\{A_{\tau}\right\}+\mathfrak{N}+\mathfrak{J}$ is invertible if and only if (17) is invertible for all $m \in \mathbf{R} \cup\{\infty\}$ and that the invertibility of $S_{1}\left\{A_{\tau}\right\}+\mathcal{K}$ is equivalent to the invertibility of (18) for all $m \in \mathbf{R} \cup\{\infty\}$. This completes the proof of (15) and thus the proof of Theorem 8.1.

Theorem 8.2 can be proved in a similar fashion. Again,

$$
\begin{aligned}
& \mathfrak{J}=\left\{\left\{A_{\tau}\right\}+\mathfrak{N} \in \mathfrak{D}_{a}:\right. \\
&\left.\qquad A_{\tau}=P_{\tau} K P_{\tau}+R_{\tau} L R_{\tau} \text { with compact } K \text { and } L\right\}
\end{aligned}
$$

is a closed two-sided ideal of $\mathfrak{D}_{a}$, and Proposition 5.1 holds with $\mathfrak{D}_{a}$ in place of $\mathfrak{A}$. Define $\mathcal{D}_{a}$ as the smallest $C^{*}$-subalgebra of $\mathcal{L}\left(L^{2}(0, \infty)\right)$ containing the operator $W(a)$ and all operators $W(c)$ with $c \in C$. The mapping

$$
\delta: \mathfrak{D}_{a} / \mathfrak{J} \rightarrow \mathcal{D} / \mathcal{K}, \quad\left\{A_{\tau}\right\}+\mathfrak{N}+\mathfrak{J} \mapsto S_{1}\left\{A_{\tau}\right\}+\mathcal{K}
$$

is a $C^{*}$-algebra homomorphism of $\mathfrak{D}_{a} / \mathfrak{J}$ onto $\mathcal{D}_{a} / \mathcal{K}$. Localizing in $\mathfrak{D}_{a} / \mathfrak{J}$ and $\mathcal{D}_{a} / \mathcal{K}$ over the same central subalgebras $(\mathfrak{A}+\mathfrak{J}) / \mathfrak{J}$ and $\mathcal{B} / \mathcal{K}$ as in the $P C$ case gives that

$$
\left(\mathfrak{D}_{a} / \mathfrak{J}\right) / \mathfrak{Y}_{m} \quad \text { and } \quad\left(\mathcal{D}_{a} / \mathcal{K}\right) / \mathcal{Y}_{m}
$$

are isometrically isomorphic to the $C^{*}$-algebra of all continuous functions on

$$
\Lambda_{0}\left(\left\{W_{\tau}(a)\right\}+\mathfrak{N}+\mathfrak{J}+\mathfrak{Y}_{m}\right) \quad \text { and } \quad \Lambda_{0}\left(W(a)+\mathcal{K}+\mathcal{Y}_{m}\right)
$$

respectively. The determination of the spectra (20) is possible due to the happy circumstance that these two spectra depend only on the "local range" $\mathcal{R}_{m}(a)$ and that $\mathcal{R}_{m}(a)$ lies entirely on some line segment. The arguments of the proofs of Theorem 4.67 and of Section 7.31(c) of [6] show that both spectra (20) are equal to the closed convex hull conv $\mathcal{R}_{m}(a)$ of $\mathcal{R}_{m}(a)$. Finally, as in the $P C$ case, we obtain that

$$
\left\{A_{\tau}\right\}+\mathfrak{N}+\mathfrak{J}+\mathfrak{Y}_{m} \quad \text { and } \quad S_{1}\left\{A_{\tau}\right\}+\mathcal{K}+\mathfrak{Y}_{m}
$$

are represented by the same function in $C\left(\operatorname{conv} \mathcal{R}_{m}(a)\right)$, which in the end implies that $\delta$ is an isometric isomorphism of $\mathfrak{D}_{a} / \mathfrak{J}$ onto $\mathcal{D} / \mathcal{K}$. 
After that we see that Proposition 5.3 and Theorem 3.4 are valid with $\mathfrak{D}_{a}$ in place of $\mathfrak{A}$, and this completes the proof of Theorem 8.2.

We remark that the algebras $\mathfrak{D}_{a} / \mathfrak{J}$ and $\mathcal{D}_{a} / \mathcal{K}$ are both commutative. Indeed, since the algebras (19) are isomorphic to the commutative $C^{*}$ algebra $C\left(\operatorname{conv} \mathcal{R}_{m}(a)\right)$, it follows that the algebras (19) themselves are commutative. Hence,

$$
\left\{W_{\tau}(a) W_{\tau}(\bar{a})-W_{\tau}(\bar{a}) W_{\tau}(a)\right\}+\mathfrak{N}+\mathfrak{J} \in \mathfrak{Y}_{m}
$$

and

$$
W(a) W(\bar{a})-W(\bar{a}) W(a)+\mathcal{K} \in \mathcal{Y}_{m}
$$

for all $m \in N$, and since $\cap_{m \in N} Y_{m}$ is the zero ideal in $R$ (this is also part of the general local principle of Allan and Douglas, see $[\mathbf{1 1}]$ or $[\mathbf{6}]$ ), it follows that

$$
\left\{W_{\tau}(a) W_{\tau}(\bar{a})-W_{\tau}(\bar{a}) W_{\tau}(a)\right\} \in \mathfrak{N}+\mathfrak{J}
$$

and

$$
W(a) W(\bar{a})-W(\bar{a}) W(a) \in \mathcal{K},
$$

which proves the commutativity of $\mathfrak{D}_{a} / \mathfrak{J}$ and $\mathcal{D} / K$. In other words, if $a \in L^{\infty}(\mathbf{R})$ is locally normal, then $W(a)$ and $W(\bar{a})$ commute modulo compact operators, while $W_{\tau}(a)$ and $W_{\tau}(\bar{a})$ commute modulo operators of the form $P_{\tau} K P_{\tau}+R_{\tau} L R_{\tau}+C_{\tau}$ with compact operators $K$ and $L$ (independent of $\tau$ ) and operators $C_{\tau}$ for which $\left\|C_{\tau}\right\| \rightarrow 0$ as $\tau \rightarrow \infty$.

9. Discrete convolutions and Toeplitz operators. All the problems, methods, and results discussed in the preceding eight sections have their discrete analogues, and in fact much more is known for discrete convolution operators than for their integral counterparts. The central problem is to relate the spectral properties of a discrete convolution operator $T_{n}$ given on $l^{2}$ over $\mathbf{Z}_{0, n}=\{k \in \mathbf{Z}: 0 \leq k \leq n\}$ by

$$
\left(T_{n} \varphi\right)_{i}=\sum_{j=0}^{n} a_{i-j} \varphi_{j}, \quad 0 \leq i \leq n
$$

to the spectral properties of the discrete convolution operator $T$ defined on $l^{2}$ over $\mathbf{Z}_{+}=\{k \in \mathbf{Z}: 0 \leq k\}$ as

$$
(T \varphi)_{i}=\sum_{j=0}^{\infty} a_{i-j} \varphi_{j}, \quad 0 \leq i
$$


or to those of the discrete convolution operator $L$ acting on $l^{2}$ over all of $\mathbf{Z}$ by the rule

$$
(L \varphi)_{i}=\sum_{j=-\infty}^{\infty} a_{i-j} \varphi_{j}, \quad-\infty<i<\infty
$$

The appropriate language is the language of the Toeplitz and Laurent operators and matrices.

For a function $a$ in $L^{\infty}$ on the complex unit circle $\mathbf{T}$, denote by $\left\{a_{n}\right\}_{n \in \mathbf{Z}}$ the sequence of its Fourier coefficients:

$$
a\left(e^{i \theta}\right)=\sum_{n \in \mathbf{Z}} a_{n} e^{i n \theta}, \quad e^{i \theta} \in \mathbf{T}
$$

It is the boundedness of the function $a$ which implies that the infinite matrices $\left(a_{i-j}\right)_{i, j=0}^{\infty}$ and $\left(a_{i-j}\right)_{i, j=-\infty}^{\infty}$ induce bounded operators on $l^{2}\left(\mathbf{Z}_{+}\right)$and $l^{2}(\mathbf{Z})$, which are called the Toeplitz and the Laurent operator with the symbol $a$ and are denoted by $T(a)$ and $L(a)$, respectively. For $a \in L^{\infty}(\mathbf{T})$ and $n \in \mathbf{Z}_{+}$, the truncated (Toeplitz) operator $T_{n}(a)$ is given by the matrix $\left(a_{i-j}\right)_{i, j=0}^{n}$ on $l^{2}\left(\mathbf{Z}_{0, n}\right)=\mathbf{C}^{n+1}$.

Given a sequence $\left\{E_{n}\right\}_{n=0}^{\infty}$ of sets $E_{n} \subset \mathbf{C}$, we denote by $\lim _{n \rightarrow \infty} E_{n}$ the set of all $\lambda \in \mathbf{C}$ for which there exist $n_{1}, n_{2}, \ldots$ and $\lambda_{1}, \lambda_{2}, \ldots$ such that

$$
0<n_{1}<n_{2}<\cdots, \quad n_{k} \rightarrow \infty, \quad \lambda_{k} \in E_{n k}, \quad \lambda_{k} \rightarrow \lambda
$$

From the work of Szegö $[\mathbf{2 2}]$ and Widom [24] we know that if $a \in L^{\infty}(\mathbf{T})$ is real-valued, then

$$
\lim _{n \rightarrow \infty} \Lambda_{0}\left(T_{n}(a)\right)=\Lambda_{0}(T(a))=[\underset{t \in \mathbf{T}}{\operatorname{essinf}} a(t), \underset{t \in \mathbf{T}}{\operatorname{esssup}} a(t)],
$$

and since the spectrum $\Lambda_{0}(L(a))$ is the essential range $\mathcal{R}(a)$ of $a$, it is clear that (21) is not true with $\Lambda_{0}(T(a))$ replaced by $\Lambda_{0}(L(a))$. In case $a \in L^{\infty}(\mathbf{T})$ is rational, the limit set $\lim _{n \rightarrow \infty} \Lambda_{0}\left(T_{n}(a)\right)$ was identified by Schmidt and Spitzer [19] and Day [9]: it turns out that this limit set is a "thin" set (a finite union of analytic arcs) contained in $\Lambda_{0}(T(a)$ ) and "trying to stay away" from the boundary of $\Lambda_{0}(T(a))$. 
For symbols $a \in L^{\infty}(\mathbf{T})$ that are neither rational nor real-valued, nice discussions of the problem are in $[\mathbf{2 7}]$ and $[\mathbf{2}]$. In particular, with respect to the question of whether $\Lambda_{0}\left(T_{n}(a)\right)$ mimics $\Lambda_{0}(T(a))$ or $\Lambda_{0}(M(a))$, Basor and Morrison [2] write: "Obviously, these are conflicting pictures and the truth is a mixture of the two, with the eigenvalues approaching $\Lambda_{0}(L(a))$ by staying in the interior of $\Lambda_{0}(T(a))$ as can be seen from plots of actual examples. This behavior is typical but it has not been rigorously proved for a large class of symbols."

A function $a \in L^{\infty}(\mathbf{T})$ is said to be piecewise continuous or to belong to $P C$ if the one-sided limits $a\left(e^{i(\theta \pm 0)}\right)$ exist at each $e^{i \theta} \in \mathbf{T}$. The Toeplitz operator $T(a)$ induced by the piecewise continuous function

$$
a\left(e^{i \theta}\right)=\pi i e^{i \theta / 2}, \quad 0 \leq \theta<2 \pi
$$

is given by the Hilbert matrix $(1 /(i-j-1 / 2))_{i, j=0}^{\infty}$ and is a wonderful example of a symbol that is neither rational nor real-valued. It is shown in [27] and [2] (also see [5] and [15]) that if $a$ is given by (22), then $\lim _{n \rightarrow \infty} \Lambda_{0}\left(T_{n}(a)\right)$ equals $\Lambda_{0}(L(a))$ and is not (!) equal to $\Lambda_{0}(T(a))$, which underpins the above quotation from $[\mathbf{2}]$.

But now suppose $a \in P C$ is the function which equals 1 on the upper and -1 on the lower half circle. Then $a$ is real-valued and (21) tells us that $\lim _{n \rightarrow \infty} \Lambda_{0}\left(T_{n}(a)\right)=[-1,1]$, whereas the Basor-Morrison quotation suggests that the limit set has rather to do with the set $\Lambda_{0}(L(a))=\{-1,1\}$ !? The enigma's resolution is that although for large $n$ the eigenvalues of $T_{n}(a)$ are tightly distributed throughout the interval $[-1,1]$, most of them lie near -1 or 1 . To put this into precise language, let $\mu_{n}$ denote the measure on $[-1,1]$ given by

$$
\mu_{n}(E)=\frac{1}{n+1} \times \text { number of eigenvalues of } T_{n}(a) \text { in } E \text {; }
$$

Szegö [22] showed that if $f$ is any continuous function on $[-1,1]$, then

$$
\begin{aligned}
\lim _{n \rightarrow \infty} \frac{1}{n+1} \sum_{\lambda \in \Lambda_{0}\left(T_{n}(a)\right)} f(\lambda) & =\int_{-1}^{1} f(\lambda) d \mu_{n}(\lambda) \\
& =\frac{f(-1)+f(1)}{2} \\
& =\int_{-1}^{1} f(\lambda) d \mu(\lambda),
\end{aligned}
$$


where $\mu(E)$ is half of the cardinality of $\{-1,1\} \cap E$. Equivalently, $\mu_{n}$ converges weakly to the probability measure concentrated at $\{-1,1\}$, and in this sense Basor and Morrison are completely right.

Let us now turn to the discrete analogues of the main results established in the previous sections.

For $a \in L^{\infty}(\mathbf{T})$ and an open arc $U \subset \mathbf{T}$, the essential range $\mathcal{R}_{U}(a)$ is defined as the spectrum of the restriction $a \mid U$ in $L^{\infty}(U)$. A function $a \in L^{\infty}(\mathbf{T})$ is called locally normal if for each $t \in \mathbf{T}$ the set

$$
\mathcal{R}_{t}(a)=\bigcap_{t \in U} \mathcal{R}_{U}(a)
$$

the intersection over all open $\operatorname{arcs} U \subset \mathbf{T}$ containing $t$, lies on some straight line segment (depending on $t$ ).

Theorem 9.1. Let $a \in L^{\infty}(\mathbf{T})$ be locally normal. If $T(a)$ is invertible, then $T_{n}(a)$ is invertible for all sufficiently large $n$, the limit $\lim _{n \rightarrow \infty}\left\|T_{n}^{-1}(a)\right\|$ exists, and

$$
\lim _{n \rightarrow \infty}\left\|T_{n}^{-1}(a)\right\|=\left\|T^{-1}(a)\right\| .
$$

This result is very implicity already contained in [21].

The proof is completely analogous to the one we have given for the Wiener-Hopf case: the role of $\mathfrak{F}$ is now played by the set of all sequences $\left\{A_{n}\right\}_{n=0}^{\infty}$ of operators $A_{n} \in \mathcal{L}\left(l^{2}\left(\mathbf{Z}_{0, n}\right)\right)$ such that

$$
\left\|\left\{A_{n}\right\}\right\|:=\sup _{n \geq 0}\left\|A_{n}\right\|<\infty
$$

instead of the operators $P_{\tau}$ and $R_{\tau}$ we have to work with $P_{n}$ and $R_{n}$ given by

$$
\begin{aligned}
& P_{n}:\left(\varphi_{0}, \varphi_{1}, \varphi_{2}, \ldots\right) \mapsto\left(\varphi_{0}, \varphi_{1}, \ldots, \varphi_{n}, 0,0, \ldots\right), \\
& R_{n}:\left(\varphi_{0}, \varphi_{1}, \varphi_{2}, \ldots\right) \mapsto\left(\overline{\varphi_{n}}, \overline{\varphi_{n-1}}, \ldots, \overline{\varphi_{0}}, 0,0, \ldots\right),
\end{aligned}
$$

and so on.

As in the Wiener-Hopf case, we can prove the following results. 
Theorem 9.2. If $a \in L^{\infty}(\mathbf{T})$ is locally normal and $\varepsilon>0$, then

$$
\lim _{n \rightarrow \infty} \Lambda_{\varepsilon}\left(T_{n}(a)\right)=\Lambda_{\varepsilon}(T(a)) .
$$

A theorem like this first appeared in Reichel and Trefethen's paper [18], where it is derived from the results of Widom [26].

Theorem 9.3. Let $a \in L^{\infty}(\mathbf{T})$ be locally normal, and let $f: \mathbf{R} \rightarrow \mathbf{C}$ be any continuous function. Then

$$
\lim _{n \rightarrow \infty} \Lambda_{0}\left(f\left(T_{n}(\bar{a}) T_{n}(a)\right)=\Lambda_{0}(f(T(\bar{a}) T(a))) \cup \Lambda_{0}(f(T(a) T(\bar{a}))),\right.
$$

where $\bar{a}\left(e^{i \theta}\right)=\overline{a\left(e^{i \theta}\right)}$.

Theorems of this type were first established by Parter [16], Widom [26] and Silbermann [21].

We finally remark that Theorems 8.1 and 8.2 (and thus the assertions of Examples 7.2, 7.4 and 7.5) can be completely carried over to the Toeplitz case.

Acknowledgments. I am greatly indebted to Harold Widom for his valuable comments on an earlier draft of this paper. I also wish to thank Harald Heidler, who proved a version of Proposition 6.1 that implied the validity of Proposition 6.2 for all sufficiently small $\varepsilon>0$. Last, but not least, I am very grateful to Andrzej Daniluk for communicating to me the proof of Proposition 6.1 given in the text.

\section{REFERENCES}

1. P.M. Anselone and I.H. Sloan, Spectral approximations for Wiener-Hopf operators II, J. Integral Equations Appl. 4 (1992), 465-489.

2. E. Basor and K.E. Morrison, The Fisher-Hartwig conjecture and Toeplitz eigenvalues, Linear and Multilinear Algebra, to appear.

3. A. Böttcher, Analysis lokal sektorieller Matrixfunktionen und geometrische Spektraltheorie von Toeplitzoperatoren, Habilitationsschrift, TU Karl-Marx-Stadt, 1987.

4. A. Böttcher and B. Silbermann, The finite section method for Toeplitz operators on the quarter-plane with piecewise continuous symbols, Math. Nachr. 11 (1983), 279-291. 
5. - Toeplitz operators and determinants generated by symbols with one Fisher-Hartwig singularity, Math. Nachr. 127 (1986), 95-124.

6. - Analysis of Toeplitz operators, Springer-Verlag, Berlin, Heidelberg, New York, 1990.

7. A. Böttcher and $\mathrm{H}$. Widom, Two remarks on spectral approximations for Wiener-Hopf operators, J. Integral Equations Appl. 6 (1994), 31-36.

8. A. Brown and P. Halmos, Algebraic properties of Toeplitz operators, J. reine angew. Math. 213 (1963), 89-102.

9. K.M. Day, Measures associated with Toeplitz matrices generated by Laurent expansion of rational functions, Trans. Amer. Math. Soc. 209 (1975), 175-183.

10. J. Dixmier, Les $C^{*}$-algèbres et leurs représentations, Gauthier-Villars, Paris, 1969.

11. R.G. Douglas, Banach algebra techniques in operator theory, Academic Press, New York, 1972.

12. R. Duduchava, Integral equations with fixed singularities, Teubner, Leipzig, 1979 .

13. I. Gohberg and I.A. Feldman, Convolution equations and projection methods for their solution, Amer. Math. Soc., Providence, RI, 1974 (Russian original: Nauka, Moscow, 1971; German translation: Akademie-Verlag, Berlin, 1974).

14. I. Gohberg and N. Ya. Krupnik, On the algebra generated by Toeplitz matrics, Funct. Anal. Appl. 3 (1969), 119-127.

15. R.A. Libby, Asymptotics of determinants and eigenvalue distributions of Toeplitz matrices with certain discontinuous symbols, Ph.D. thesis, University of California, Santa Cruz, 1990.

16. S.V. Parter, On the distribution of the singular values of Toeplitz matrices, Linear Algebra Appl. 80 (1986), 115-130.

17. S.C. Reddy, Pseudospectra of Wiener-Hopf integral operators and constantcoefficient differential operators, J. Integral Equations Appl. 5 (1993), 369-403.

18. L. Reichel and L.N. Trefethen, Eigenvalues and pseudo-eigenvalues of Toeplitz matrices, Linear Algebra Appl. 162 (1992), 153-185.

19. P. Schmidt and F. Spitzer, The Toeplitz matrices of an arbitrary Laurent polynomial, Math. Scand. 8 (1960), 15-38.

20. B. Silbermann, Lokale Theorie des Reduktionsverfahrens für Toeplitzoperatoren, Math. Nachr. 104 (1982), 45-56.

21. - On the limiting set of singular values of Toeplitz matrices, Linear Algebra Appl. 182 (1993), 35-43.

22. G. Szegö, Beiträge zur Theorie der Toeplitzschen Formen I, Math. Z. 6 (1920), 167-202

23. S.R. Treil, The invertibility of a Toeplitz operator does not imply its invertibility by the projection method, Sov. Math. Dokl. 35 (1987), 103-107.

24. H. Widom, Toeplitz matrices, Studies in Real and Complex Analysis (I.I. Hirschman, Jr., ed.), MAA Stud. Math. 3 (1965), 179-209.

25. - Asymptotic behavior of block Toeplitz matrices and determinants II, Adv. Math. 21 (1976), 1-29. 
26. - On the singular values of Toeplitz matrices, Z. Anal. Anwendungen 8 (1989), $221-229$.

27. - Eigenvalue distribution of nonselfadjoint Toeplitz matrices and the asymptotics of Toeplitz determinants in the case of nonvanishing index, Oper. Theory Adv. Appl. 48 (1990), 387-421.

Technische Universität Chemnitz-Zwickau, Fakultät für Mathematik, PSF 964, D-09009 Chemnitz, Germany 